\title{
2 \\ ON SPECIFYING SERVICES AND SYNTHESIZING PROTOCOLS FOR REAL-TIME APPLICATIONS
}

\author{
A. Khoumsi* , G.v. Bochmann and R. Dssouli \\ Université de Montréal, DIRO, C.P. 6128, Succursale A \\ Montréal, (Quebec), H3C 3J7
}

\begin{abstract}
This paper deals with synthesizing automatically the specifications of the protocol and of the medium, from the specification of a desired service and the model of the medium containing timing requirements. Firstly, we propose a detailed model for specifying a desired service to be provided by a real-time discrete event system. This model uses a global clock, and several fictitious timers and counters. Next, we propose a method for deriving protocol and medium specifications from service specifications for real-time applications. Such derived specifications use a different model which does not use counters. Compared to [KBD93], the application field is much broader, because two important restrictions are removed. Firstly, temporal requirements are between events which are not necessarily consecutive. Secondly, the systems considered can be concurrent .
\end{abstract}

KEY WORDS : desired service, protocol synthesis, timing requirements, supremal model of the medium, sequential and concurrent systems, timed automata, untimed automata, temporized automata.

\section{Introduction}

A discrete event system (DES) is a dynamic system where events are executed instantaneously, causing a discrete change of the state of the system. If sequences of events are a regular language, the system can be specified by a finite automaton. A first example of DES is a telecommunication network; an event can then be the transmission of a packet of data. Another example is a communication protocol, and an event can be execution of a service primitive. For some DESs it is not enough to specify the ordering of events, we must also specify temporal requirements between events. This class of DESs are called Real-time DESs. For specifying a desired service to be provided by real-time DES, we use timed automata (TA) which are defined by using a global clock, and several fictitious timers and counters. And for synthesizing the protocol which provides such desired service, we use the approach which consists of transforming a realtime problem to an untimed problem ([AD90,BW92]). In comparison with [KBD93], two extensions are made: (a) temporal requirements are not only between consecutive events; (b) concurrent systems are considered. Henceforth, DES means Real-time discrete event system.

This paper is organized as follows. In Section 2, we introduce the problem of the protocol derivation. The basic principle used for deriving the protocol is explained. In Section 3, we introduce in detail the model developed for specifying a desired service and for defining a supremal model of the medium. This model is based on timed automata which are defined in this section. In Section 4, we present the approach which consists of transforming timed automata into untimed automata which are FSMs containing a transition tick. In Section 5, we present the approach which consists of transforming untimed automata into temporized automata. Let's notice that timed and temporized automata are based on different models. In Section 6, we propose a method for deriving automatically the specifications of the protocol and of timing contraints on the medium (temporized automata), from the specification of a desired service and the supremal model of the medium (timed automata). Both sequential and parallel systems are considered. And at last, we conclude in Section 7. We will notice that the possible concurrency in the parallel systems, and the timing requirements cause a problem of state space explosion and of complexity.

\section{Problem of the protocol derivation of real-time systems}

In a real-time distributed system (RTDS, Fig.1), $n$ protocol entities (with $n>1$ ) communicate : (a) with the user of the system through several service access points (SAP); (b) with each other through a medium assumed reliable. To each SAP corresponds one protocol entity.

In the user's viewpoint, the RTDS is a black box where only interactions with the user are visible. These interactions correspond to the executions of service primitives (or simply primitives). Therefore, the specification of the service desired by (or provided to) the user defines the ordering and timing requirements between the executed primitives.

But in the designer's viewpoint, it is necessary to compute the specifications of the local real-time protocol entities $\mathrm{PE}_{\mathrm{i}}$, for $\mathrm{i}=1,2, \ldots, \mathrm{n}$, which may provide the service desired by the user. The designer must also compute timing requirements which must be respected by the medium. In order to avoid the computation of timing requirements impossible to respect by the medium, the designer may refer to a supremal model of the medium, and compute only timing requirements which respect this supremal model. Informally, if for instance we know that the medium needs at least two units of clock time (uct) to

\footnotetext{
* Supported by FCAR-NSERC-BNR grant
} 
carry messages between two protocol entities, this information is contained in the supremal model. In this case, the designer will not compute timing requirements such as : some message must be carried in one uct. We will see that the medium not only carries a message, but it also adds an information about the transit delay of the message in the medium.

The problem for desigining protocols is then : how can we derive systematically the different local protocol specifications and the timing requirements on the medium, from : (a) a global specification of the service desired by the user; (b) a supremal model of the medium.
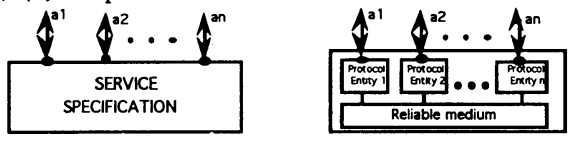

Figure 1. Service and protocol concepts

The approach used for deriving protocols is synthesis ([BG86, KBK89, SP90, KHB92, KBD93]). Timing requirements are considered in [KBD93], but they are only between consecutive events, and the systems considered are sequential. In the present study, these two constraints are removed. For the sake of simplicity, we explain the basic principle of protocol derivation only for sequential systems. But parallel systems also are considered, farther in this paper (Section 6.3). The principle is then : if a primitive $\mathrm{A}$ is executed by a protocol entity $\mathrm{PE}_{\mathrm{a}}$, and is followed by execution of a primitive $\mathrm{B}$ by $\mathrm{PE}_{\mathrm{b}}$, then after execution of $A$ by $P_{a}$, this one sends a message to $P_{b}$ to inform it that it may execute $B$. If after execution of $A$ by $P E_{a}$, there is a choice between several primitives executed by different $P E_{b i}$, for $i=1,2, \ldots$, $\mathrm{p}$, then $\mathrm{PE}_{\mathrm{a}}$ selects one $\mathrm{PE}_{\mathrm{bi}}$ and sends a message to it to inform it that it may execute one of its primitives. Let's notice that that the terms "derivation" and "synthesis" are used as synonyms.

\section{Model of the desired service and of the medium}

The desired service and the supremal model of the medium are the two starting points of the protocol derivation. For specifying them, we use a global digital clock which generates a fictitious event tick at a constant frequency ([Os90, BW92, OW90]). The delay between two ticks represents the passing of one unit of clock time (uct). The set $\mathbb{N}$ of natural numbers is our domain of time. The time is then modeled by a global variable, noted $\tau$ and called discrete time $: \tau$ is initially equal to zero and is incremented by one after the passing of each unit of clock time (uct), i.e., after the occurrence of a tick .

\subsection{Timed traces and Timed languages}

A finite timed trace trc over an alphabet $\mathrm{V}$ is a finite sequence of pairs $\left\langle\sigma_{\mathrm{i}}, \tau_{\mathrm{i}}\right\rangle$, where $\sigma_{\mathrm{i}}$ is an event of $\mathrm{V}$, and $\tau_{i}$ is an integer such that $\tau_{i+1} \geq \tau_{i}$. Such trace is represented by trc $=\left\langle\sigma_{1}, \tau_{1}\right\rangle \ldots\left\langle\sigma_{n}, \tau_{n}\right\rangle$ and contains all events that have occurred before time $\tau_{n}+1$. Each $\left\langle\sigma_{i}, \tau_{i}\right\rangle$ means that the event $\sigma_{i}$ has occurred when the discrete time is equal to $\tau_{i}$. It is clear that there is an inaccuracy of one uct on the exact delay of event occurrences. An infinite timed trace Trc over an alphabet $\mathrm{V}$ is an infinite sequence of pairs $\left\langle\sigma_{\mathrm{i}}, \tau_{\mathrm{i}}\right\rangle$; any finite prefix of Trc is called a finite timed trace over V. Such infinite trace is represented by $\operatorname{Trc}=$ $\left\langle\sigma_{1}, \tau_{1}\right\rangle \ldots\left\langle\sigma_{i}, \tau_{i}\right\rangle \ldots$ Each pair $\left\langle\sigma_{i}, \tau_{\mathrm{i}}\right\rangle$ defined in a trace Trc is called a component of this trace and is noted : $\left\langle\sigma_{\mathrm{i}}, \tau_{\mathrm{i}}\right\rangle \in \operatorname{Trc}$. Since a $\tau_{\mathrm{i}}$ may be equal to $\tau_{\mathrm{i}+1}$, several consecutive events may occur at the same discrete time, i.e., during one uct.

Definition 1. (Finiteness property)

An infinite timed trace respects the finiteness property (FP) if the number of events executed during one uct is bounded by an arbitrary constant Mc. Formally, $\operatorname{Trc}=\left\langle\sigma_{1}, \tau_{1}\right\rangle \ldots\left\langle\sigma_{i}, \tau_{i}\right\rangle \ldots$ respects the FP if and only if : $\forall \mathrm{i}>0, \exists \mathrm{j}>\mathrm{i}$ such that $\tau_{\mathrm{j}-1}=\tau_{\mathrm{i}}<\tau_{\mathrm{j}}$ and $\mathrm{j} \leq \mathrm{i}+\mathrm{Mc}$. The FP is differently defined in [TH92], where it only requires that a finite number of events occur in any finite time interval.

Example 1. (a) Let $\operatorname{Trc}$ be the following infinite trace $\operatorname{Trc}=\left\langle\sigma_{1}, 2\right\rangle\left\langle\sigma_{2}, 4\right\rangle \ldots\left\langle\sigma_{\mathrm{i}}, 2 \mathrm{i}\right\rangle \ldots$ Trc respects the finiteness property because one event occurs when $\tau$ is even, and no event occurs when $\tau$ is odd.

(b) Let $\operatorname{Trc}$ be the following infinite trace $\operatorname{Trc}=\left\langle\sigma_{1}, 1\right\rangle^{1}\left\langle\sigma_{2}, 4\right\rangle^{2} \ldots\left\langle\sigma_{\mathrm{i}}, 2 \mathrm{i}\right\rangle^{\mathrm{i}} \ldots$, where $\langle\sigma, \tau\rangle \mathrm{P}$ means that $\sigma$ occurs p times when the discrete time is equal to $\tau$. Trc does not respect the FP because the number of events during one uct is not bounded.

Definition 2. (Timed trace and timed language)

In this paper, we consider only infinite timed traces. Such traces, will be simply called timed traces. A timed language $\mathcal{L}$ over an alphabet $\mathrm{V}$ is a set of infinite timed traces over $\mathrm{V}$.

We say that $\mathcal{L}$ respects the finiteness property (FP) if all its timed traces respect the FP. 
Infinite timed traces, which will be simply called timed traces, are executed by non terminating processes. This is not really a restriction. In fact, a terminating process which may be executed infinitely often, can also be considered as a non terminating process.

Definition 3. (Projection of a timed trace)

Let $\mathrm{V}$ be a subset of an alphabet $\mathrm{W}$, and let $\operatorname{Trc}=\left\langle\sigma_{1}, \tau_{1}\right\rangle \ldots\left\langle\sigma_{\mathrm{i}}, \tau_{\mathrm{i}}\right\rangle \ldots$ be a timed trace over $\mathrm{W}$. The projection of $\operatorname{Trc}$ on $\mathrm{V}$, noted Projv(Trc), is obtained by removing from $\operatorname{Trc}$ all $\left\langle\sigma_{\mathrm{i}}, \tau_{\mathrm{i}}\right\rangle$, where $\sigma_{\mathrm{i}} \notin \mathrm{V}$.

Definition 4. (Projection and Extension of a timed language)

Let $\mathrm{V}$ be a subset of an alphabet $\mathrm{W}$. Let $\mathcal{L}_{1}$ be a timed language over $\mathrm{W}$. The projection of $\mathcal{L}_{1}$ on $\mathrm{V}$, noted $\operatorname{Projv}\left(\mathcal{L}_{1}\right)$, is defined by : $\operatorname{Projv}\left(\mathcal{L}_{1}\right)=\left\{\operatorname{Trc}\right.$, over V| $\exists \operatorname{Trce} \in L_{1}$ with $\left.\operatorname{Trc}=\operatorname{Projv}(\operatorname{Trce})\right\}$; Let $L_{2}$ be a timed language over $\mathrm{V}$. The extension of $L_{2}$ to $\mathrm{W}$, noted $\operatorname{Ext}_{\mathrm{W}}\left(\mathcal{L}_{2}\right)$, is defined by :

$\operatorname{Ext}_{\mathrm{w}}\left(\mathcal{L}_{2}\right)=\left\{\operatorname{Trc}\right.$, over W IProjv $\left.(\operatorname{Trc}) \in L_{2}\right\}$.

Remark 1. (a) if $\mathrm{W}=\mathrm{V}$ then $\operatorname{Projv}(\mathcal{L})=\operatorname{Ext} \mathrm{W}(\mathcal{L})=\mathcal{L} ;($ b) $\operatorname{Projv}(\operatorname{Extw}(\mathcal{L}))=\mathcal{L}$ and $\mathcal{L} \subseteq \operatorname{Extw}(\operatorname{Projv}(\mathcal{L}))$.

3.2. Timers and counters

A DES may be specified by a timed automaton, or simply a TA, which is an extended FSM accepting a timed language (Def. 2). For defining a TA, we use several fictitious timers and counters.

Definition 5. (Timer)

A fictitious timer $T_{i}$ is a conceptual entity associated to a variable $t_{i}$ belonging to the set $\mathbb{N}$ of natural numbers. $\mathrm{t}_{\mathrm{i}}$ is automatically incremented after the passing of one uct, and is called the current value of timer $T_{j}$. The operations we can do on the timer are :

- Reset : a timer $T_{i}$, whose value $t_{i}$ is increasing regularly by one after each uct, can be set to zero. $t_{i}$ represents therefore the time elapsed from the last reset of timer $T_{i}$.

- Comparison : the value $t_{i}$ of timer $T_{i}$ can be compared to a constant integer. The comparison operators are $=,>$ and $\leq$. Other operators $<$ and $\geq$ are not necessary because timer values are integers.

Initially, when the discrete time $\tau$ is equal to zero, $\mathrm{t}_{\mathrm{i}}$ also is equal to zero.

We deduce that if several timers $T_{1}, T_{2}, \ldots, T_{N}$ are used, then their current values $t_{1}, t_{2}, \ldots, t_{N t}$ are automatically and simultaneously incremented after the passing of one uct, i.e. when the discrete time $\tau$ is incremented. Therefore, all the timers are synchronized on the digital global clock.

Definition 6. (Timer state)

Let $\mathrm{Nt}$ (or $|\mathrm{T}|$ ) be the number of timers $\mathrm{T}_{1}, \mathrm{~T}_{2}, \ldots, \mathrm{T}_{\mathrm{Nt}}$. The Nt-uplet $\mathrm{ts}_{\mathrm{s}}=\left(\mathrm{t}_{1}, \ldots, \mathrm{t}_{\mathrm{N}}\right)$, where $\mathrm{t}_{\mathrm{i}}$ is the current value of timer $T_{i}$, is called the current timer state .

Definition 7. ( $\mathrm{T}$ _Condition, set $\mathrm{E}_{\mathrm{T}}$ )

Let $T=\left\{T_{1}, T_{2}, \ldots, T_{N t}\right\}$ be a set of timers. A $T_{-}$Condition $E(t s)$, w.r.t. $T$, is a boolean function depending on the current timer state $t s=\left(t_{1}, \ldots, t_{N}\right)$. $E(t s)$ is formed from : (a) canonical boolean functions $t_{i} \sim k$, where

$t_{i}$ is the current value of a timer $T_{i}, k \in \mathbb{N}^{*}$, and $\sim$ is $=\leq$ or $>$;

(b) operators $\operatorname{AND}(\wedge), \operatorname{OR}(\vee)$, and NOT $(\neg)$ on these canonical boolean functions.

The set of all T_Conditions, w.r.t. T, is noted $\mathrm{E}_{\mathrm{T}}$.

Definition 8. (Counter) A fictitious counter $C_{i}$, w.r.t. an alphabet $V c_{i}$, is a conceptual entity associated to a variable $c_{i}$ belonging to $\mathbb{N}$. $c_{i}$ is called the current value of $C_{i}$, and is automatically : (a) incremented after the occurrence of any event of $\mathrm{Vc}_{\mathrm{i}}$; (b) set to zero after the passing of one uct, i.e.,when $\tau$ is incremented.

Definition 9. (counter state) Let $\mathrm{Nc}$ (or $\mid \mathrm{Cl}$ ) be the number of counters $\mathrm{C}_{1}, \mathrm{C}_{2}, \ldots, \mathrm{C}_{\mathrm{Nc}}$. The Nc-uplet $c s=\left(c_{1}, \ldots, c_{N c}\right)$, where $c_{i}$ is the current value of counter $C_{i}$, is called the current counter state .

Definition 10. (F_Condition, set $E_{C}$ ) Let $C=\left\{C_{1}, C_{2}, \ldots, C_{N c}\right\}$ be a set of counters. A F_Condition $K(c s)$, w.r.t. $\mathrm{C}$, is a boolean function depending on the current counter state $\mathrm{cs}=\left(\mathrm{c}_{1}, \ldots, \mathrm{c}_{\mathrm{Nc}}\right) . \mathrm{K}$ (cs) is formed from

: (a) canonical boolean functions $c_{i}<M c_{i}$, where $c_{i}$ is the current value of a counter $C_{i}$, and $M c_{i} \in \mathbb{N}^{*}$; (b) operator $\operatorname{AND}(\wedge)$ on these canonical functions. The set of all F_Conditions, w.r.t. C, is noted $\mathrm{E}_{\mathrm{C}}$.

Example 2. if $\mathrm{K}(\mathrm{cs})=\left(\mathrm{c}_{1}<M c_{1}\right)$ must be always true, and $\mathrm{C}_{1}$ is w.r.t. $\mathrm{Vc}_{1}$, then no more than $\mathrm{Mc}_{1}$ events of $\mathrm{Vc}_{1}$ may occur during one uct.

3.3 Timed Automata for real-time processes

For defining a TA, we use in general:

- a global digital clock which informs about the passing of one uct,

- a finite set of fictitious digital timers (Def. 5), for specifying the timing requirements,

- a finite set of counters (Def. 8), for respecting the finiteness property (Def. 1). 
Definition 11. (Timed transition, and Reset)

Let $A=\left(Q, V, \delta, q_{0}\right)$ be a FSM where $Q$ is a set of states, $V$ is an alphabet, $q_{0}$ is the initial state, and $\delta \subseteq Q \times V \times Q$ defines the transitions, i.e., a transition of $A$ can be represented by $\left[q_{1} ; \sigma ; q_{2}\right]$.

Let $T=\left\{T_{1}, \ldots, T_{N t}\right\}$ be a set of timers, and let $C=\left\{C_{1}, \ldots, C_{N c}\right\}$ be a set of counters, w.r.t $V c_{i} \subseteq V$, for $\mathrm{i}=1,2, \ldots, \mathrm{N}_{\mathrm{c}}$. Let $\mathrm{E}_{\mathrm{T}}$ (resp. $\mathrm{E}_{\mathrm{C}}$ ) be the sets of $\mathrm{T}_{-}$Conditions (resp. F_Conditions), w.r.t. T (resp. C).

A timed transition, w.r.t. $\mathrm{T}$ and $\mathrm{C}$, is defined by $\mathrm{Tr}=\left[\mathrm{q}_{1} ; \sigma ; \mathrm{q}_{2} ; \mathrm{E}(\mathrm{ts}) ; \mathrm{R} ; \mathrm{K}\right.$ (cs)], with $\sigma \in \mathrm{V}, \mathrm{q}_{1}, \mathrm{q}_{2} \in \mathrm{Q}$, $\mathrm{E}(\mathrm{ts}) \in \mathrm{E}_{\mathrm{T}}, \mathrm{K}(\mathrm{cs}) \in \mathrm{E}_{\mathrm{C}}$, and $\mathrm{R} \subseteq \mathrm{T}$. $\mathrm{R}$ is called Reset of the transition $\mathrm{Tr}$. The semantics of $\mathrm{Tr}$ is the following. Let $q_{1}$ be the current state : (1) $\sigma$ may occur only if $\mathrm{E}(\mathrm{ts})$ (Def.7) and $\mathrm{K}$ (cs) (Def.10) are true;

(2) after the occurrence of $\sigma:$ (a) the state $\mathrm{q}_{2}$ is reached, timers of $\mathrm{R}$ are set to zero, and

(b) $c_{i}$ is incremented if $\sigma \in \mathrm{Vc}_{\mathrm{i}}$, for $\mathrm{i}=1,2, \ldots, \mathrm{N}_{\mathrm{c}}$.

Besides, $\mathrm{K}(\mathrm{cs})=\left(\mathrm{c}_{\mathrm{i} 1}<\mathrm{Mc_{11 }}\right) \wedge . . \wedge\left(\mathrm{c}_{\mathrm{ip}}<\mathrm{M} \mathrm{c}_{\mathrm{ip}}\right)$, where $\mathrm{c}_{\mathrm{i} 1}, \ldots, \mathrm{c}_{\mathrm{ip}}$ are all counters respectively w.r.t. $\mathrm{V}_{\mathrm{i} 1}, \ldots, \mathrm{V}_{\mathrm{ip}}$,

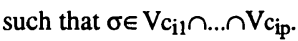

Informally, the event $\sigma$ of $\mathrm{V}$ in $\mathrm{Tr}=\left[\mathrm{q}_{1} ; \sigma ; \mathrm{q}_{2} ; \mathrm{E}(\mathrm{ts}) ; \mathrm{R} ; \mathrm{K}(\mathrm{cs})\right]$ may occur only if the T_Condition $\mathrm{E}(\mathrm{ts})$ is true. Besides, if $\sigma \in \mathrm{V}_{\mathrm{c}_{i}}(\subseteq \mathrm{V})$, then $\left(\mathrm{c}_{\mathrm{i}}<\mathrm{Mc_{i }}\right)$ also must be true for occurrence of $\sigma$.

Definition 12. (Enabled and Eligible timed transition)

A timed transition $\left[q_{1} ; \sigma ; q_{2} ; \mathrm{E}(\mathrm{ts}) ; \mathrm{R} ; \mathrm{K}(\mathrm{cs})\right]$ is enabled if conditions for occurrence of $\sigma$ are true (Def.11).

A timed transition $\operatorname{Tr}=\left[\mathrm{q}_{1} ; \sigma ; \mathrm{q}_{2} ; \mathrm{E}(\mathrm{ts}) ; \mathrm{R} ; \mathrm{K}(\mathrm{cs})\right]$ is eligible if :

$\operatorname{Tr}$ is enabled or will become enabled with the passing of time (without occurrence of any event).

A timed automaton (TA) $A^{t}$ can then be constructed from the FSM $A=\left(Q, V, \delta, q_{0}\right.$ ), the finite sets $T$ (of timers $T_{1}, \ldots, T_{N}$ ) and $C$ (of counters $C_{1}, \ldots, C_{N c}$ ). For that, we transform each transition $t r=\left[q_{1} ; \sigma ; q_{2}\right]$ of $A$ into a timed transition $\operatorname{Tr}$ (Def.11) by associating to it, a T_condition $\mathrm{E}(\mathrm{ts})$, a Reset, and a F_Condition.

In this paper, we consider only TA which accept (Def.14) a timed language, i.e., infinite timed traces.

Definition 13. (Timed automaton)

A timed automaton $\mathrm{A}^{\mathrm{L}}=\left(\mathrm{Q}, \mathrm{V}, \mathrm{T}, \mathcal{V}, \delta, \mathrm{q}_{0}\right)$ is defined as follows. $\mathrm{Q}$ is the set of states, $\mathrm{q}_{0}$ is the initial state,

$V$ is the alphabet, $T$ is the set of timers $T_{1}, T_{2}, \ldots, T_{N t}, V=\left\{V c_{i} \mid\right.$ for $\left.i=1,2, \ldots, N c\right\} \subset 2^{V}$, where each $V c_{i}$ is associated to one counter $C_{i}$, and $\delta \subseteq Q \times V \times Q \times E_{T} \times 2^{T} \times E_{C}$ defines the timed transitions (Def.11), where $E_{T}$ and $E_{C}$ are the sets of $T_{-}$Conditions and $F_{-}$Conditions (Def.7 and 10).

Besides, $\mathrm{A}^{\mathrm{t}}$ accepts only infinite timed traces (Def. 14), and is called a sequential TA.

Examples of timed automata are given in Section 3.6. (Exemple 3).

Remark 2. Let's notice that the timers and counters are fictitious. For example, the desired service of Figure 7 just means that the user wants that there must be at most two uct between primitives $A_{1}$ and $B_{2}$. But the timers do not really exist. Therefore, a question such as, how can we use a same timer in a distributed system, has no sense. If the timers were real, there would be a problem of using a same timer in different sites.

Definition 14. (Acceptance of a timed trace and of a language, equivalence, partial order relation)

Let $A^{t}$ be a $T A\left(Q, V, T, \mathcal{V}, \delta, q_{0}\right)$ and let $\operatorname{Trc}=\left\langle\sigma_{1}, \tau_{1}\right\rangle \ldots\left\langle\sigma_{i}, \tau_{i}\right\rangle \ldots$ be an infinite timed trace.

- Trc is accepted by $\mathrm{A}^{\mathrm{t}}$, is formally defined by :

$\forall \mathrm{i} \in \mathbb{N}^{*}: \exists \operatorname{Tr}_{\mathrm{i}}=\left[\mathrm{q}_{\mathrm{i}-1} ; \alpha_{\mathrm{i}} ; \mathrm{q}_{\mathrm{i}} ; \mathrm{E}_{\mathrm{i}}(\mathrm{ts}) ; \mathrm{R}_{\mathrm{i}} ; \mathrm{K}_{\mathbf{i}}(\mathrm{cs})\right] \in \delta$ with $:\left(\alpha_{\mathrm{i}}=\sigma_{\mathfrak{i}}\right) \wedge\left(\left(\tau=\tau_{\mathrm{i}}\right) \Rightarrow\left(\mathrm{E}_{\mathrm{i}}(\mathrm{ts}) \wedge \mathrm{K}_{\mathrm{i}}(\mathrm{cs})=\mathrm{True}\right)\right)$.

Informally, a system specified by $A^{t}$ may execute a trace accepted by $A^{t}$.

- A timed language, noted $\mathcal{L}_{\mathrm{A}}$ t is accepted by $\mathrm{A}^{\mathrm{t}}$ if it contains all and only the traces accepted by $\mathrm{A}^{\mathrm{t}}$.

- $A_{1}^{t}$ and $A_{2}^{t}$ are equivalent, and noted $A_{1}^{t} \cong A_{2}^{t}$, if and only if $\mathcal{L}_{A_{1}^{t}}=\mathcal{L}_{A_{2}^{t}}$.

- $A_{1}^{t}$ is smaller than or equal to $A_{2}^{t}$, and noted $A_{1}^{t} \leq A_{2}^{t}$, if and only if $L_{A_{1}^{t}} \subseteq L_{A_{2}^{t}}$.

Property 1. Let $A^{t}=\left(Q, V, T, \mathcal{V}, \delta, q_{0}\right)$ be a timed automaton specifying a non terminating system, with $V=\left\{V_{c_{1}}, V c_{2}, \ldots, V c_{N c}\right\} 2^{V}$. If $V c_{1} \cup \ldots \cup V c_{N c}=V$, then the language $\mathcal{L}_{A} t$ accepted by $A^{t}$ (Def. 14) respects the finiteness property. In this case, we say that $\mathrm{A}^{\mathrm{t}}$ respects the finiteness property.

Definition 15. (set $\tau$ of timer states)

Let $T=\left\{T_{1}, \ldots, T_{N t}\right\}$ be a set of timers used for defining a $T A A^{t}$, and let $M_{i}$ be the maximum value a timer $T_{i}$ is compared to, for defining the $T_{-}$Conditions (Def.7) of all the transitions of $A^{t}$. In this case, the value $t_{i}$ of $T_{i}$ does not need to be incremented as soon as $t_{i}=M_{i}+1$. In fact, in this case the 
incrementation would have no influence on truths of the T_Conditions. Therefore, we can limit the value of $t_{i}$ by $M t_{i}+1$, for $i=1,2, \ldots, N t$, and the set $\tau$ of timer states $t s=\left(t_{1}, \ldots, t_{N}\right)$ is equal to or included in $\left\langle 0 ; \mathrm{Mt}_{1}+1\right) \times \ldots \times\left\langle 0 ; \mathrm{Mt}_{\mathrm{Nt}}+1\right\rangle$, where $\left\langle 0 ; \mathrm{Mt}_{\mathrm{i}}+1\right\rangle$ is the set of integers belonging to the interval $\left[0 ; \mathrm{Mt}_{\mathrm{i}}+1\right]$.

For the TA of Fig.2.a (Exemple 3), Mt $1_{1}=5$, and $M t 1_{2}=5$, and then $\tau \subseteq\langle 0 ; 6\rangle \times\langle 0 ; 6\rangle$

Definition 16. (Addition between $\tau$ and $\mathbb{N}$ )

Let $T=\left\{T_{1}, \ldots, T_{N t}\right\}$ be a set of timers used for defining a $T A A^{t}$. The addition between $\tau$ and $\mathbb{N}$ is defined as follows : if $t_{s}=\left(t_{1}, \ldots, t_{N}\right) \in \mathcal{T}$ and $p \in \mathbb{N}$, then $t s+p=\left(\inf \left(t_{1}+p, M t_{1}+1\right), \ldots, \inf \left(t_{N i}+p, M_{N_{N}}+1\right)\right)$.

Where inf is defined by : $\inf (A, B) \in\{A, B\}$ and $((\inf (A, B)=A) \Leftrightarrow(A<B))$.

Intuitively, if ts is the current timer state, then $t s+p$ is the futur timer state after the passing of $p$ units of clock time (uct). For the TA of Fig. 2 .a, if $t s=(4,1)$ and $p=3$, then $t s+3=(\inf (4+3 ; 6), \inf (1+3 ; 6))=(6,4) \neq(7,4)$.

Definition 17. (set $C$ of counter states)

Let $C=\left\{C_{1}, \ldots, C_{N c}\right\}$ be a set of counters used for defining a TA $A^{t}$, and let $M c_{i}$ be the maximum value which bounds the value $c_{i}$. Therefore, the set $C$ of counter states $c s=\left(c_{1}, \ldots, \mathrm{cNc}_{\mathrm{Nc}}\right)$ is equal to or included in $\left\langle 0 ; \mathrm{Mc}_{1}\right\rangle \times \ldots \times\left\langle 0 ; \mathrm{Mc}_{\mathrm{Nc}}\right\rangle$, where $\left\langle 0 ; \mathrm{Mc}_{\mathrm{i}}\right\rangle$ is the set of integers belonging to the interval $\left[0 ; \mathrm{Mt}_{\mathrm{i}}\right]$.

\subsection{Product of two timed automata over the same alphabet}

Let $A_{1}^{t}$ and $A_{2}^{t}$ be two TA (Def.13) defined over the same alphabet $V$. An intuitive definition of the synchronized product of $A_{1}^{t}$ and $A_{2}^{t}$, noted $A_{1}^{t} \times A_{2}^{t}$, is the following: $A_{1}^{t} \times A_{2}^{t}$ is a TA specifying a system which may execute all and only the infinite timed traces accepted by both $A_{1}^{t}$ and $A_{2}^{t}$.

Definition 18. (Product over a same alphabet)

Let $A_{i}^{t}=\left(Q i, V, T i, \mathcal{Z}, \delta i, q i_{0}\right)$, for $i=1,2$, be two $T A$ over a same alphabet $V$, with $T 1 \cap T 2=\varnothing$, and $\mathcal{U}=\left\{\mathrm{Vci}_{1}, \ldots, \mathrm{Vci}_{\mathrm{Nc}_{\mathrm{i}}}\right\}$. Each $\mathrm{A}_{\mathrm{i}}^{t}$ uses then a set $\mathrm{Ti}=\left\{\mathrm{Ti}_{1}, \ldots, \mathrm{Ti}_{\mathrm{Nti}_{\mathrm{i}}}\right\}$ of timers and a set $\mathrm{Ci}=\left\{\mathrm{Ci}_{1}, \ldots, \mathrm{Ci}_{\mathrm{NC}_{\mathrm{i}}}\right\}$ of counters, where each $C_{j}$ is w.r.t. $V c_{j}$. The product, noted $A^{t}=A_{1}^{t} \times A_{2}^{t}$, is defined by $A^{t}=\left(Q, V, T, \mathcal{V}, \delta, q_{0}\right)$, with $\mathcal{V}=\mathcal{V} \backslash \mathcal{V} 2, \mathrm{~T}=\mathrm{T} 1 \cup \mathrm{T} 2, \mathrm{Q} \subseteq \mathrm{Q} 1 \times \mathrm{Q} 2, \mathrm{q}_{0}=\left\langle\mathrm{q}_{1}, \mathrm{q} 2_{0}\right\rangle \in \mathrm{Q}$, and :

Definition of $\delta$ : Let $\mathrm{E}_{\mathrm{T} 1}, \mathrm{E}_{\mathrm{T} 2}$ and $\mathrm{E}_{\mathrm{T}}$ be the set of T_Conditions (Def. 7), respectively w.r.t. $\mathrm{T} 1, \mathrm{~T} 2$ and $\mathrm{T}=\mathrm{T} 1 \cup \mathrm{T} 2$. Let $\mathrm{E}_{\mathrm{C}_{1}}, \mathrm{E}_{\mathrm{C} 2}$ and $\mathrm{E}_{\mathrm{C}}$ be the set of $\mathrm{F}_{-}$Conditions (Def. 10), respectively w.r.t. $\mathrm{C} 1, \mathrm{C}_{2}$ and $\mathrm{C}=\mathrm{C} 1 \cup \mathrm{C} 2$. Then $\forall\langle\mathrm{q} 1, \mathrm{q} 2\rangle,\langle\mathrm{r} 1, \mathrm{r} 2\rangle \in \mathrm{Q}, \forall \sigma \in \mathrm{V}, \forall \mathrm{E} \in \mathrm{E}_{\mathrm{T}}, \forall \mathrm{R} \subseteq \mathrm{T}, \forall \mathrm{K} \in \mathrm{E}_{\mathrm{C}}$ :

$([\langle\mathrm{q} 1, \mathrm{q} 2\rangle, \sigma,\langle\mathrm{r} 1, \mathrm{r} 2\rangle, \mathrm{E}, \mathrm{R}, \mathrm{K}] \in \delta) \Leftrightarrow\left(\exists \mathrm{E}_{1} \in \mathrm{E}_{\mathrm{T} 1}, \exists \mathrm{E}_{2} \in \mathrm{E}_{\mathrm{T} 2}, \exists \mathrm{R} 1 \subseteq \mathrm{T} 1, \exists \mathrm{R} 2 \subseteq \mathrm{T} 2, \exists \mathrm{K}_{1} \in \mathrm{E}_{\mathrm{Cl}}, \exists \mathrm{K}_{2} \in \mathrm{E}_{\mathrm{C} 2},\right)$

(with: $\mathrm{R}=\mathrm{R} 1 \cup \mathrm{R} 2, \mathrm{E}=\mathrm{E} 1 \wedge \mathrm{E} 2, \mathrm{~K}=\mathrm{K} 1 \wedge \mathrm{K}_{2}$, and

$([q 1, \sigma, \mathrm{r} 1, \mathrm{E} 1, \mathrm{R} 1, \mathrm{~K} 1] \in \delta 1$, and $[\mathrm{q} 2, \sigma, \mathrm{R} 2, \mathrm{E} 2, \mathrm{R} 2, \mathrm{~K} 2] \in \delta 2$. )

Theorem 1. If $L_{A_{1}^{t}}$ and $L_{A_{2}^{t}}$ are respectively the timed languages accepted by $A_{1}^{t}$ and $A_{2}^{t}$ over the same

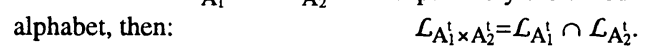

Property 2. In Def. 18, if $\mathrm{Vc}_{1} \cup \ldots \cup V \mathrm{Vc}_{\mathrm{Nc}_{1}}=\mathrm{Vc2}{ }_{1} \cup \ldots \cup V \mathrm{Vc}_{2} \mathrm{Nc}_{2}=\mathrm{V}$, then $A_{1}^{\mathrm{t}}, A_{2}^{\mathrm{t}}$, and $A_{1}^{\mathrm{t}} \times A_{2}^{t}$ respect the finiteness property.

Remark3. (a) In Def.18, if there exist $i \leq N c_{i}$ and $j \leq N c_{j}$ such that $V c 1_{i}=V c 2_{j}$, then counters $C_{1} 1_{i}$ and $C 2_{j}$ may be a same counter for defining $A_{1}^{t}, A_{2}^{t}$, and $A_{1}^{t} \times A_{2}^{t}$. In fact, the values $c 1_{i}$ and $c 2_{j}$ are incremented and set to zero simultaneously. Therefore, one counter, for example $\mathrm{Cl}_{\mathbf{i}}$, is sufficient.

(b) From Theorem 1, we deduce that if $A_{1}^{t}$ and $A_{2}^{t}$ specify two sequential processes over the same alphabet, then their synchronized product also specifies a sequential process.

3.5. Product of two timed automata over alphabets $V_{1}$ and $V_{2}$ with $V_{1} \subseteq V_{2}$

Before defining the product over alphabets $V_{1}$ and $V_{2}$, with $V_{1} \subseteq V_{2}$, let's give two definitions.

Definition 19. (Operator $\oplus$ on En)

Let $E_{1}(t s), E_{2}(t s), \ldots, E_{k}(t s)$ be $k T$ Tonditions (Def. 7), depending on a set of timers $\left\{T_{1}, T_{2}, \ldots, T_{N t}\right\}$. We define $\mathrm{E}(\mathrm{ts})=\mathrm{E}_{1}(\mathrm{ts}) \oplus \mathrm{E}_{2}(\mathrm{ts}) \oplus \ldots \oplus \mathrm{E}_{\mathrm{k}}(\mathrm{ts})$ as follows.

$$
(\mathrm{E}(\mathrm{ts})=\text { false }) \Leftrightarrow\left\{\forall \mathrm{i} \in\{1, \ldots, \mathrm{k}\}, \forall \mathrm{p} \in \mathbb{N}: \mathrm{E}_{\mathrm{i}}(\mathrm{ts}+\mathrm{p})=\text { false }\right\}
$$

Informally, $\mathrm{E}_{1}(\mathrm{ts}) \oplus \ldots \oplus \mathrm{E}_{\mathbf{k}}(\mathrm{ts})$ is false if and only if all $\mathrm{E}_{\mathrm{i}}(\mathrm{ts})$ are false and remain false with the passing of time. If for instance $\mathrm{T}=\left\{\mathrm{T}_{1}\right\}, \mathrm{E}_{1}\left(\mathrm{t}_{1}\right)=\left(\mathrm{t}_{1} \leq 5\right), \mathrm{E}_{2}\left(\mathrm{t}_{1}\right)=\left(\left(\mathrm{t}_{1}>2\right) \Lambda\left(\mathrm{t}_{1} \leq 6\right)\right)$, then $\mathrm{E}\left(\mathrm{t}_{1}\right)=\mathrm{E}_{1}\left(\mathrm{t}_{1}\right) \oplus \mathrm{E}_{2}\left(\mathrm{t}_{1}\right)=\left(\mathrm{t}_{1} \leq 6\right)$. 
Definition 20. (Extension of a timed automaton)

Let $A^{t}=\left(Q, V, T, \mathcal{V}, \delta, q_{0}\right)$ be a $T A$ over an alphabet $V$ with $T=\left\{T_{1}, \ldots, T_{N t}\right\}, V=\left\{V c_{1}, \ldots, V c_{N c}\right\}$, and then $C=\left\{C_{1}, \ldots, C_{N c}\right\}$. Let $E_{T}$ (resp. $E_{C}$ ) be the set of T_Conditions w.r.t. T (resp. F_Conditions w.r.t. C). Let W be an alphabet such that $\mathrm{V} \subseteq \mathrm{W}$. The extension of $\mathrm{A}^{t}$ to the alphabet $\mathrm{W}$, noted $\operatorname{Ext}\left(\mathrm{A}^{t}\right)$, is a TA defined by $\left(\mathrm{Q}, \mathrm{W}, \mathrm{T}, \mathcal{V}, \delta_{\text {ext }}, \mathrm{q}_{0}\right)$, where $\delta_{\mathrm{ext}} \subseteq \mathrm{Q} \times \mathrm{W} \times \mathrm{Q} \times \mathrm{E}_{\mathrm{T}} \times 2^{\mathrm{T}} \times \mathrm{E}_{\mathrm{C}}$ is such that :

(1) $\forall \mathrm{q} 1, \mathrm{q} 2 \in \mathrm{Q}, \forall \sigma \in \mathrm{V}, \forall \mathrm{E} \in \mathrm{E}_{\mathrm{T}}, \forall \mathrm{R} \subseteq \mathrm{T}, \forall \mathrm{K} \in \mathrm{E}_{\mathrm{C}}:\left[\langle\mathrm{q} 1, \sigma, \mathrm{q} 2, \mathrm{E}, \mathrm{R}, \mathrm{K}] \in \delta \Leftrightarrow\left[\langle\mathrm{q} 1, \sigma, \mathrm{q} 2, \mathrm{E}, \mathrm{R}, \mathrm{K}] \in \delta_{\text {ext }}\right.\right.$.

(2) $\forall \mathrm{q} \in \mathrm{Q}$ : Let $\mathrm{E}_{\mathrm{i}} \in \mathrm{E}_{\mathrm{T}}$, for $\mathrm{i}=1, \ldots, \mathrm{k}$, be all the T_Conditions of $\mathrm{E}_{\mathrm{T}}$ such that : $\exists q_{\mathrm{i}} \in \mathrm{Q}, \exists \sigma_{\mathrm{i}} \in \mathrm{V}$, $\exists R_{i} \in 2^{T}, \exists K_{i} \in E_{C}$, with $\left[q, \sigma_{i}, q_{i}, E_{i}, R_{i}, K_{i}\right] \in \delta$, and let then $E=E_{1} \oplus E_{2} \oplus \ldots \oplus E_{k}$.

Then $\forall \sigma \in W-V:\left[\left\langle q, \sigma, q^{\prime}, E^{\prime}, R, K\right] \in \delta_{\text {ext }} \Leftrightarrow\left(q^{\prime}=q, E^{\prime}=E, R=\varnothing, K=T r u e\right)\right.$.

If $\mathrm{B}^{\mathrm{t}}=\operatorname{Ext} \mathrm{t}_{\mathrm{W}}\left(\mathrm{A}^{\mathrm{t}}\right)$, then $\mathrm{A}^{\mathrm{t}}$ is called projection of $\mathrm{B}^{t}$ in the alphabet $\mathrm{V}$, and is noted $\left.\mathrm{A}^{\mathrm{t}}=\operatorname{Proj} \mathrm{B}^{\mathrm{t}}\right)$.

Informally, $\operatorname{Ext}_{\mathrm{W}}\left(\mathrm{A}^{\mathrm{t}}\right)$ is obtained by adding selfloops of all events of $\mathrm{W}-\mathrm{V}$ to each state of $\mathrm{A}^{\mathrm{t}}$. The resets of these selfloops are empty, and their T_conditions are defined as follows. The T_Condition of the added selfloops at a state $\mathrm{q}$ of $\mathrm{A}^{\mathrm{t}}$ is true if at least one of the transitions defined in $\mathrm{A}^{t}$ from $\mathrm{q}$ is eligible. The F_Condition for events of W-V is always true, and then $\operatorname{Ext}_{w}\left(\mathrm{~A}^{t}\right)$ does not necessarily respect the finiteness property (Property 1 ).

Lemme 1. If $\mathcal{L}_{\mathrm{A}}$ is the timed language accepted by a TA $\mathrm{A}^{\mathrm{t}}$ over an alphabet $\mathrm{V}$, and if $\mathrm{W}$ is an alphabet such that $V_{\subseteq}$, then : $\mathcal{L}_{\operatorname{Ext}}\left(A^{t}=\operatorname{Ext}_{W}\left(\mathcal{L}_{A^{t}}\right)\right.$. (see Def. 4 for $\operatorname{Ext}_{W}\left(\mathcal{L}_{A^{t}}\right)$ )

Before defining formally the product over $V 1$ and $V 2$ with $V 1 \subseteq V 2$, let's give an intuitive definition. Let $A_{1}^{t}$ and $A_{2}^{t}$ be two TA defined over $V_{1}$ and $V_{2}$ with $V_{1} \subseteq V_{2}$. The product of these two TA is a TA specifying a system which may execute all and only the infinite timed traces which both:

are accepted by $A_{2}^{t}$, and whose projections (Def. 3) on V1 are accepted by $A_{1}^{t}$.

Definition 21. (Product over $\mathrm{V} 1$ and $\mathrm{V} 2$ with $\mathrm{V} 1 \subseteq \mathrm{V} 2$ )

Let $A_{i}^{t}=\left(Q i, V i, T i, \mathcal{H}, \delta i, q i_{0}\right)$, for $i=1,2$, be two TA (Def.13) over alphabets $V 1$ and V2, with V1ᄃV2, $\mathrm{T} 1 \cap \mathrm{T} 2=\varnothing$, and $\mathcal{U}=\left\{\mathrm{Vci}_{1}, \ldots, \mathrm{Vci}_{\mathrm{Nci}}\right\}$, i.e., each $A_{i}^{t}$ uses a set $\mathrm{Ci}=\left\{\mathrm{Ci}_{1}, \ldots, \mathrm{Ci}_{\mathrm{NC}_{\mathrm{i}}}\right\}$ of counters where each $\mathrm{Ci}_{\mathrm{j}}$ is w.r.t. Vcij. Their synchronized product, noted $A_{1}^{t} \otimes A_{2}^{t}$, is defined by :

$A_{1}^{t} \otimes A_{2}^{t}=\left(Q, V 2, T 1 \cup T 2, \mathcal{V} \cup \mathcal{V}, \delta, q_{0}\right)=\operatorname{Ext}_{V 2}\left(A_{1}^{t}\right) \times A_{2}^{t} \quad\left(\right.$ See Def.18 and 20 for $\times$ and $\left.\operatorname{Ext}_{V 2}\left(A_{1}^{t}\right)\right)$.

Theorem 2. If $\mathcal{L}_{\mathrm{A}_{1}^{t}}$ and $\mathcal{L}_{\mathrm{A}_{2}^{t}}$ are respectively the timed languages accepted by $\mathrm{A}_{1}^{t}$ and $\mathrm{A}_{2}^{t}$ respectively over alphabets $V_{1}$ and $V 2$, with $V 1 \subseteq V 2$, then: $\mathcal{L}_{\mathrm{A}_{1}^{\mathrm{t}} \otimes \mathrm{A}_{2}^{\mathrm{t}}}=\mathcal{L} \operatorname{Ext}_{2}\left(\mathrm{~A}_{1}^{\mathrm{t}}\right) \cap \mathcal{L}_{\mathrm{A}_{2}^{\mathrm{t}}}$.

Property 3. Let $A_{1}^{t}$ and $A_{2}^{t}$ be two TA, respectively over alphabets $V 1$ and $V 2$ with $V 1 \subseteq V 2$. If $A_{2}^{t}$ respects the finiteness property (FP), then $A_{1}^{t} \otimes A_{2}^{t}$ respects the FP.

Remark4. (a) in Definition 21, if $V_{1}=V_{2}$, then $A_{1}^{t} \otimes A_{2}^{t}=A_{1}^{t} \times A_{2}^{t}$ (Def. 18), because $\operatorname{Ext}_{2}\left(A_{1}^{t}\right)=A_{1}^{t}$;

(b) From Theorem 2, we deduce that if $A_{1}^{t}$ and $A_{2}^{t}$ specify two sequential processes respectively over alphabets $\mathrm{V} 1$ and $\mathrm{V} 2$ with $\mathrm{V} 1 \subseteq \mathrm{V} 2$, then their synchronized product also specifies a sequential process.

\subsection{General parallel product of two timed automata}

Before defining formally the parallel product of two TA $A_{1}^{t}$ and $A_{2}^{t}$, respectively over alphabets $V_{1}$ and $V 2$, let's give an intuitive definition. The product of $A_{1}^{t}$ and $A_{2}^{t}$ is a TA specifying a parallel system which may execute all and only the timed traces over the alphabet V1UV2: (a) whose projections (Def.3) on V1 are accepted (Def.14) by $A_{1}^{t}$ and ; (b) whose projections on $V 2$ are accepted by $A_{2}^{t}$.

Definition 22. (Parallel product of two TA)

Let $A_{i}^{t}=\left(Q i, V i, T i, \mathcal{U}, \delta i, q i_{0}\right)$, for $i=1,2$, be two $T A$ over alphabets $V 1$ and $V 2$, with $T 1 \cap T 2=\varnothing$, and $\mathcal{H}=\left\{\mathrm{Vci}_{1}, \ldots, \mathrm{Vci}_{\mathrm{NC}_{\mathrm{i}}}\right\}$. Their parallel product, noted $\mathrm{A}_{1}^{\mathrm{t}} \| \mathrm{A}_{2}^{\mathrm{t}}$, is defined by :

$A_{1}^{t} \| A_{2}^{t}=\left(Q, V 1 \cup V 2, T 1 \cup T 2, \mathcal{U} \cup V_{2}, \delta, q_{0}\right)=\operatorname{Ext} V_{1} \cup V_{2}\left(A_{1}^{t}\right) \times \operatorname{Ext}_{2} \cup V_{1}\left(A_{2}^{t}\right)$.

Remark 5. In Definition 22, if $V_{1} \subseteq V_{2}$ then $A_{1}^{t} \| A_{2}^{t}=A_{1}^{t} \otimes A_{2}^{t}, \quad$ and if $V_{1}=V_{2}$ then $A_{1}^{t} \| A_{2}^{t}=A_{1}^{t} \times A_{2}^{t}$ 
Theorem 3. If $\mathcal{L}_{\mathrm{A}_{1}^{t}}$ and $\mathcal{L}_{\mathrm{A}_{2}^{t}}$ are the timed languages accepted by two $\mathrm{TA} \mathrm{A}_{1}^{\mathrm{t}}$ and $\mathrm{A}_{2}^{\mathrm{t}}$ over alphabets $\mathrm{V}_{1}$

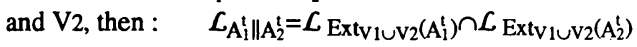

Property 4. If two TA $A_{1}^{t}$ and $A_{2}^{t}$, respectively over alphabets $V_{1}$ and $V 2$, respect the finiteness property, then $A_{1}^{t} \| A_{2}^{t}$ respects the finiteness property.

Example 3. Let $A_{1}^{t}=\left(Q 1, V 1, T 1, \mathcal{U}, \delta 1, q 1_{0}\right)$ and $A_{2}^{t}=\left(Q 2, V 2, T 2, V_{2}, \delta 2, q 2_{0}\right)$ (Figure 2$)$, with $\mathcal{U}=\left\{V i_{1}\right\}=\{V i\}$, $\mathrm{Qi}=\left\{\mathrm{qi}_{0}, \mathrm{qi}\right\}, \mathrm{Ti}=\left\{\mathrm{Ti}_{1}, \mathrm{Ti}_{2}\right\}, \mathrm{Mc}=\mathrm{Mci}_{1}=10$, for $\mathrm{i}=1,2 . \mathrm{V} 1=\{a, b\}, \mathrm{V} 2=\{a, c\}$. The values of timers $\mathrm{T}_{1}, \mathrm{Ti}_{2}$, $\mathrm{T}_{1}$ and $\mathrm{T} 2_{2}$, are respectively $\mathrm{t}_{1}, \mathrm{t}_{2}, \mathrm{t}_{1}$ and $\mathrm{t}_{2}$, and the values of counters $\mathrm{Cl}_{1}$ and $\mathrm{C} 2_{1}$, are respectively $\mathrm{C}_{1}$ and $\mathrm{c}_{2}$.

$\delta 1=\left\{\left[\mathrm{q} 1_{0}, a, \mathrm{q} 1, \mathrm{E} 1_{1},\left\{\mathrm{~T}_{1}\right\}, \mathrm{K} 1\right],\left[\mathrm{q} 1, b, \mathrm{q} 1_{0}, \mathrm{E} 1_{2},\left\{\mathrm{~T}_{2}\right\}, \mathrm{K} 1\right]\right\}, \mathrm{E} 1_{1}=\left(\mathrm{t1}_{1} \leq 5\right), \mathrm{E} 1_{2}=\left(\mathrm{tt}_{1} \leq 2\right) \wedge\left(\mathrm{tt}_{2} \leq 5\right), \mathrm{K} 1=\left(\mathrm{cl}_{1}<10\right)$. $\delta 2=\left\{\left[\mathrm{q} 2_{0}, a, \mathrm{q} 2, \mathrm{E} 2_{1},\left\{\mathrm{~T}_{2}\right\}, \mathrm{K} 2\right],\left[\mathrm{q} 2, c, \mathrm{q} 2_{0}, \mathrm{E} 2_{2},\left\{\mathrm{~T} 2_{2}\right\}, \mathrm{K} 2\right]\right\}, \mathrm{E} 2_{1}=\left(\mathrm{t}_{2} \leq 3\right), \mathrm{E} 2_{2}=\left(\mathrm{D}_{1}>3\right)$, and $\mathrm{K} 2=\left(\mathrm{c} 2_{1}<0\right)$.

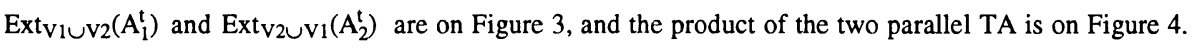
The F_Conditions (Def. 10) of transitions in $A_{1}^{t} \| A_{2}^{t}$ (Fig.4) are as follows.

Transitions with event $a$ are enabled only if both $\left(\mathrm{c1}_{1}<10\right)$ and $\left(\mathrm{c2}_{1}<\mathrm{MA}\right)$ are true $\left.\left(a \in \mathrm{Vc1}_{1} \cap \mathrm{Vc2}\right)_{1}\right)$.

Transitions with event $b$ are enabled only if $\left(\mathrm{cl}_{1}<10\right)$ is true (because $\left.b \in \mathrm{Vc1}_{1}\right)$.

Transitions with event $c$ are enabled only if $\left(\mathrm{c} 21_{1}<10\right)$ is true (because $\left.c \in \mathrm{Vc}_{2}\right)$.

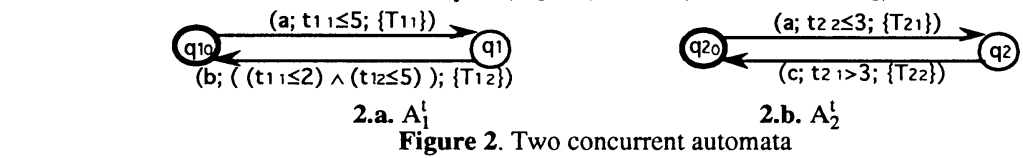

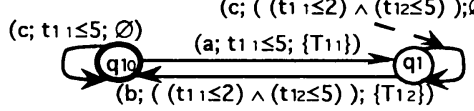

3.a. $\operatorname{Ext}_{\mathrm{V} 1 \cup \mathrm{V}_{2}}\left(A_{1}^{\mathrm{t}}\right)$

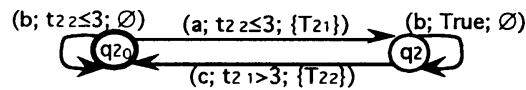

3.b. $\operatorname{Ext}_{\mathrm{V} 2 \cup \mathrm{v}_{1}}\left(\mathrm{~A}_{2}^{\mathrm{t}}\right)$

Figure 3. Extensions of the two concurrent automata of figure 3

$$
\left(b ;\left(t_{1} 1 \leq 2\right) \wedge(t 12 \leq 5) \wedge(t 2 \leq 3) ;\left\{T_{12}\right\}\right)\left(b ;\left(t_{11} \leq 2\right) \wedge\left(t t_{12} \leq 5\right) ;\left\{T_{12}\right\}\right)
$$

Figure 4. Synchronized product $A_{1}^{t} \| A_{2}^{t}$

Definition 23. (Independent and concurrent DES)

Let $A_{i}^{t}$ be two TA over alphabets $V i$, for $i=1,2$, specifying two processes.

If $V_{1} \cap V_{2}=\varnothing$, the two processes are independent with each other.

If $\mathrm{V} 1 \cap \mathrm{V} 2 \neq \varnothing$, the two processes are concurrent. In fact, they may run in parallel by executing respectively events of $V_{1}-V_{2}$ and $V_{2}-V_{1}$, but they must execute conjointly events of $V_{1} \cap V_{2}$.

\section{Untiming a timed specification}

\subsection{Untimed traces and untimed languages}

So far, an infinite sequence of events has been represented by a timed trace $\operatorname{Trc}=\left\langle\sigma_{1}, \tau_{1}\right\rangle \ldots\left\langle\sigma_{\mathrm{i}}, \tau_{\mathrm{i}}\right\rangle$...

If we use the event tick which represents the passing of one uct, the same sequence can be represented by an untimed trace TRC $=\alpha_{1} \alpha_{2} \ldots \alpha_{j} \ldots$, where each $\alpha_{j}$ for $j=1,2, \ldots$, is equal to tick or to one of $\sigma_{1}, \sigma_{2}, \ldots$

Example 4. The timed $\operatorname{Trc}=\left\langle\sigma_{1}, 2\right\rangle \ldots\left\langle\sigma_{\mathrm{i}}, 2 \mathrm{i}\right\rangle \ldots$ can equivalently be represented by the untimed :

TRC=tick tick $\sigma_{1}$ tick tick $\sigma_{2} \ldots \sigma_{\mathrm{i}-1}$ tick tick $\sigma_{\mathrm{i}}$ tick tick $\sigma_{\mathrm{i}+1} \ldots$

A formal definition of the untimed trace corresponding to a timed trace is the following.

Definition 24. (Untimed trace and operator UntimeT )

Let Trc $=\left\langle\sigma_{1}, \tau_{1}\right\rangle \ldots\left\langle\sigma_{i}, \tau_{i}\right\rangle \ldots$ be a timed trace. We define the operator UntimeT , for obtaining the untimed trace TRC corresponding to Trc, by : TRC $=$ UntimeT(Trc $)=\alpha_{1} \alpha_{2} \ldots \alpha_{j} \ldots$

with: $\quad\left(\alpha_{i+\tau_{j}}=\sigma_{i}\right)$ and $\left(\alpha_{j}=t i c k\right.$, if $\notin k>0$ such that $\left.j=k+\tau_{k}\right)$, for $i, j=1,2, \ldots$ 
If Trc respects the finiteness property (Def.1), we also say that TRC respects the finiteness property. Let's notice that the operator UntimeT is a bijection.

Property 5. Let TRC $=\alpha_{1} \alpha_{2} \ldots \alpha_{j} \ldots$ be a infinite untimed trace respecting the finiteness property. $\exists M c>0$ such that: $\forall \mathrm{k}>0, \exists \mathrm{l}_{1}>\mathrm{k}, \exists \mathrm{l}_{2}>\mathrm{k}$ with $\alpha_{11} \neq t i c k, \mathrm{l}_{2}-\mathrm{k} \leq \mathrm{Mc}+1$, and $\alpha_{12}=t i c k$.

More informally, Property 5 means that the untimed TRC corresponds to an infinite timed trace (by $\alpha_{11} \neq t i c k$ ) which respects the finiteness property (by $\mathrm{l}_{2}-\mathrm{k} \leq \mathrm{Mc}+1$ and $\alpha_{12}=$ tick).

Definition 25. (Untimed language and operator UntimeL)

Let $\mathcal{L}$ be a timed language. $L^{\mathrm{u}}$, which is called untimed language and noted $\mathcal{L}^{\mathrm{u}}=\operatorname{Untime} L(L)$, is defined by : $\quad \mathcal{L}^{\mathrm{u}}=$ Untime $L(\mathcal{L})=\{\operatorname{TRC} \mid \exists \operatorname{Trc} \in \mathcal{L}$ with $\operatorname{TRC}=$ Untime $T(\operatorname{Trc})\}$

Theorem 5. Let $\mathcal{L}_{1}$ and $\mathcal{L}_{2}$ be two timed languages over a same language. Then :

$$
\text { Untime } L\left(L_{1} \cap L_{2}\right)=\operatorname{Untime} L\left(\mathcal{L}_{2}\right) \cap \operatorname{Untime} L\left(L_{2}\right) \text {. }
$$

\subsection{Untimed automata}

Definition 26. (Untimed automaton and operator UntimeA )

Let $\mathrm{A}^{\mathrm{t}}$ be a TA over an alphabet $\mathrm{V}$ which accepts (Def. 14) a timed language $\mathcal{L}$, and let $\mathcal{L}^{\mathrm{u}}=\operatorname{Untime} L(L)$.

$A^{\text {ut }}$ is the minimal FSM over the alphabet $V \cup\{$ tick $\}$, called untimed automaton (UA) which accepts the untimed language $L^{\mathrm{u}}$. In other words :

$\mathcal{L}_{\mathrm{A}}$ ut $=$ Untime $L\left(\mathcal{L}_{\mathrm{A}} \mathrm{t}\right), \quad($ Def. 25, for UntimeL $)$

A sufficient condition of existence of $A^{\text {ut }}$ is the finiteness of the set of timers.

We also define the surjective operator UntimeA such that : $\quad \mathrm{A}^{\mathrm{ut}}=\operatorname{UntimeA}\left(\mathrm{A}^{\mathrm{t}}\right)$.

Example 5. Let's consider the timed $A^{t}=\left(Q, V, T,\{V\}, \delta, q_{0}\right)$ on Figure 5.a, where we use one timer $T_{1}$, and one counter $C_{1}$ w.r.t. $V$, with $M c_{1}=5$. In this specification, the value $c_{1}$ of $C_{1}$ is smaller than or equal to $c_{1}$, and since $\mathrm{Mt}_{1}=5$ (Def. 15), the value $t_{1}$ of $T_{1}$ is smaller than or equal to 6 . The obtained untimed $A^{\text {ut }}$ is on Figure 5.b, each state being defined by $\left\langle q, t_{1}, c_{1}\right\rangle$, where $q$ is a state in $A^{t}$.

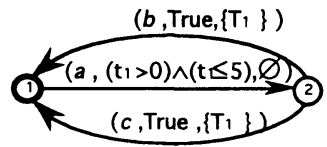

5.a. $\mathrm{A}^{\mathrm{t}}$

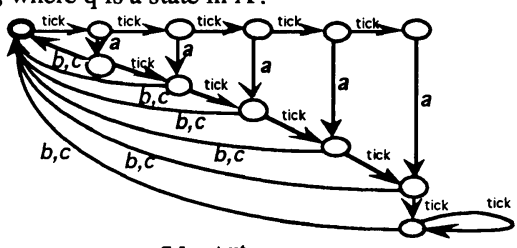

5.b. A $^{\text {ut }}$

Figure 5. Timed and untimed automata

Remark 6. Since untimed traces accepted by $A^{\text {ut }}$ correspond to infinite timed traces accepted by $A^{t}$, then $\mathrm{A}^{\text {ut }}$ accepts only infinite untimed traces, and does not contain indesirable states. An indesirable state is either a deadlock state or a state from which only a selfloop tick is executable.

- A deadlock in $\mathrm{A}^{\text {ut }}$ is indesirable, because it has no sense. In fact, a deadlock state means that the event tick is not executable. Therefore, the passing of time is stopped!

- A state from which only a selfloop tick is executable is indesirable, because it implies that $A^{\text {ut }}$ accepts a trace TRC=Untime $T(\operatorname{Trc})$ where Trc is a finite timed trace!

Informally, $\mathrm{A}^{\mathrm{ut}}$ allows to represent a real-time system specified by $\mathrm{A}^{\mathrm{t}}$, as a system without timing requirement, but where a new event tick is added. This event, which models the passing of one unit of clock time (uct), is processed like any other event.

Let's give an idea of how $A^{\text {ut }}$ is obtained from $A^{t}$ over an alphabet $V$, when only one counter $C_{1}$, w.r.t. $V c_{1}=V$ is used. This implies that $A^{t}$ respects the finiteness property (Theorem 1). Let $T=\left\{T_{1}, \ldots, T_{N t}\right\}$ be a set of timers used for defining a TA At, let $\mathrm{Mt}_{\mathrm{i}}$ be the maximum value a timer $\mathrm{T}_{\mathrm{i}}$ is compared to, for defining the T_Conditions (Def. 7) of all the transitions of $A^{t}$. In this case, the value $t_{i}$ of $T_{i}$ does not need to be incremented as soon as $\mathrm{t}_{\mathrm{i}}=\mathrm{Mt}_{\mathrm{i}}+1$ (Def. 15). A state of $\mathrm{A}^{\mathrm{ut}}$ is defined by $\left\langle\mathrm{q} 1, \mathrm{ts}, \mathrm{c}_{1}\right\rangle$, where $\mathrm{q} 1$ is a state of $A^{t}, t s=\left(t_{1}, \ldots, t_{N t}\right)$ is a timer state (Def. 6), and $c_{1}$ is a value of $C_{1}$. The passing of one uct is represented in $\mathrm{A}^{\mathrm{ut}}$ by the event tick. Execution of tick from state $\left\langle\mathrm{q} 1, t s, \mathrm{c}_{1}\right\rangle$ leads to state $\langle\mathrm{q} 1, \mathrm{ts}+1,0\rangle$, i.e., timers are incremented and the counter is set to zero. Execuition of an event $\sigma \neq t i c k$ from state $\left\langle\mathrm{q} 1, \mathrm{ts}, \mathrm{c}_{1}\right\rangle$ of $\mathrm{A}^{\mathrm{ut}}$ leads to state $\left\langle\mathrm{q} 2^{2}, \mathrm{ts}^{\prime}, \mathrm{c}_{1}+1\right\rangle$, where $\mathrm{q} 2$ is a state of $\mathrm{A}^{\mathrm{t}}$ which is reached by a transition $\operatorname{tr}=[\mathrm{q} 1, \sigma, \mathrm{q} 2, \mathrm{E}, \mathrm{R}, \mathrm{K}]$ from state 
q1 of $A^{t}$ (with $E$ true for the current timer state ts, and $c_{1}<M c_{1}$ ), and ts' is obtained from ts by setting to zero timers belonging to $R$. Besides, $A^{\text {ut }}$ is minimal.

Remark 7. (a) if $t_{s}=\left(\mathrm{Mt}_{1}+1, \ldots, \mathrm{Mt}_{\mathrm{Nt}}+1\right)$ then $\mathrm{ts}+1=\mathrm{ts}$. In this case, an event tick is a selfloop in $\mathrm{A}^{\mathrm{ut}}$; (b) Since two $A_{i}^{\text {ut }}$ over alphabets $\mathrm{V}_{\mathrm{i}} \cup\{$ tick $\}$, for $\mathrm{i}=1,2$, are $\mathrm{FSM}$, we can use the classic synchronized product between them, noted $A_{1}^{\text {ut }} \times A_{2}^{\text {ut }}$, where events of $\left(V_{1} \cap V_{2}\right) \cup\{t i c k\}$ are executed conjointly.

(c) The product UntimeA $\left(\mathrm{A}_{1}^{\mathrm{t}}\right) \times$ UntimeA $\left(\mathrm{A}_{2}^{\mathrm{t}}\right)$ may contain deadlocks, therefore it does not correspond to a real DES. In fact, a deadlock prevents the event tick, i.e., the passing of time is stopped.

Lemmes 2. Let $\mathrm{A}^{\mathrm{t}}=\mathrm{A}_{\mathrm{i}}^{\mathrm{t}}=\left(\mathrm{Q}, \mathrm{V}, \mathrm{T}, \mathcal{V}, \delta, \mathrm{q}_{0}\right)$ be a $\mathrm{TA}$ and $\mathrm{A}^{\mathrm{ut}}=\operatorname{Untime} A\left(\mathrm{~A}^{\mathrm{t}}\right)$. Let's remind some notations :

(a) $\mathrm{Nt}$ and $\mathrm{Nc}$ are the numbers of timers and counters; (b) Mc bounds all the $\mathrm{Mc}_{\mathrm{i}}$, for $\mathrm{i}=1, \ldots, \mathrm{Nc}$; (c) $\mathrm{Mt}$ is the maximum constant any timer is compared to; (d) $|Q|$ and $|\delta|$ are numbers of states and of transitions.

2.a. The number $\mid \mathrm{Qut}$ of states of $A^{\mathrm{ut}}$ is bounded by : $\quad|\mathrm{Q}| *(\mathbf{M t}+2)^{\mathbf{N t}} *(\mathbf{M c}+1)^{\mathbf{N c}}$

2.b. The number $\left|\delta^{\mathrm{ut}}\right|$ of transitions of $\mathrm{A}^{\mathrm{ut}}$ is bounded by : $(|\mathrm{Q}|+|\delta|) *(\mathbf{M t}+\mathbf{2})^{\mathbf{N t}} *(\mathbf{M c}+\mathbf{1})^{\mathbf{N c}}$

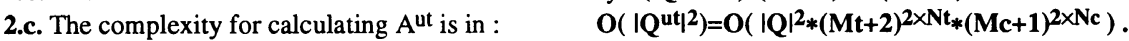
$\left|Q^{\text {ut }|,|}\right| \delta^{\text {ut } \mid}$ and the complexity for calculating $A^{\text {ut }}$ are then exponential in the numbers of timers and of counters.

Remark 8. (a) The number of counters is not really a problem. In fact, in general one counter is sufficient, for ensuring the finiteness property. Therefore, the complexity is essentially due to the number of timers. (b) If the timing requirements are only between consecutive events, one timer is sufficient for specifying temporal constraints. In this case, the complexity is no more exponential.

Properties 6. Let $A_{1}^{t}$ and $A_{2}^{t}$ be two TA respectively over alphabets $V_{1}$ and $V_{2}$.
6.a. If $\mathrm{V}_{1}=\mathrm{V}_{2}$, then :
UntimeA $\left(\mathrm{A}_{1}^{\mathrm{t}} \times \mathrm{A}_{2}^{\mathrm{t}}\right) \leq \operatorname{UntimeA}\left(\mathrm{A}_{1}^{\mathrm{t}}\right) \times \operatorname{Untime} A\left(\mathrm{~A}_{2}^{\mathrm{t}}\right)$
6.b. If $\mathrm{V}_{1} \subseteq \mathrm{V}_{2}$, then :

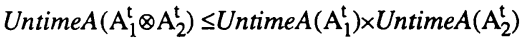

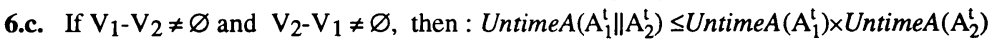
6.d. If $\mathrm{V}_{1}=\mathrm{V}_{2}$, then :
$\mathcal{L}_{\mathrm{A}_{1}^{\mathrm{t}}} \subseteq \mathcal{L}_{\mathrm{A}_{2}^{\mathrm{t}}} \Rightarrow \mathcal{L}_{\text {Untime }}\left(\mathrm{A}_{1}^{\mathrm{t}}\right) \subseteq \mathcal{L}_{\text {UntimeA }}\left(\mathrm{A}_{2}^{\mathrm{t}}\right)$
6.e. If $\mathrm{V}_{1} \subseteq \mathrm{V}_{2}$, then :
UntimeL $\left(\operatorname{Projv}_{1}\left(\mathcal{L}_{\mathrm{A}_{2}^{\mathrm{t}}}\right)\right)=\operatorname{Proj}_{1}\left(\operatorname{Untime} L\left(\mathcal{L}_{\mathrm{A}_{2}^{t}}\right)\right)$

(where $\mathrm{A} \leq \mathrm{B}$ means $\mathcal{L}_{\mathrm{A}} \mathrm{ut} \subseteq \mathcal{L}_{\mathrm{B}} \mathrm{ut}$ ).

4.3. Why untimed automata are useful.

Problem of using timed automata (TA):

(a) Respecting the timing requirements does not ensure to avoid deadlock states.

(b) Timing requirements between events are specified by using some fictitious timers. Therefore, if we project a TA into an alphabet, a few events may disappear. In this case, we have to respecify temporal requirements between events, and then we have to redefine new fictitous timers. This is not self-evident.

Interest of using untimed automata (UA) :

A UA is a FSM. Therefore, all known methods used for FSMs can be used. Let's see two examples :

(a) States respecting in general a certain "indesirable" property, in particular deadlocks states, may be removed; (b) a UA defined over an alphabet $\mathrm{W}^{\prime}=\mathrm{W} \cup\{$ tick $\}$ can be projected in any alphabet $\mathrm{V} \subseteq \mathrm{W}$ '.

Thus, before making some processings, a TA is untimed for obtaining a UA. But after the processing, it is convenient to transform the processed UA into an automaton withou tick. This is the object of Section 5.

\section{Timing untimed automata}

We propose an operator TimeA which, from a UA A $A^{\text {ut }}$, generates a temporized automaton with a new model different than the model of timed automata. A logic question arises : why two different models are used for specifying a DES ?

The first model, previously defined, is used because it is more intuitive. In the case where a TA must specify a desired behaviour, it may be constructed quite easily from an informal specification. In fact, the timers and counters are convenient fictitious entities which may be defined intuitively.

The second model is less intuitive, but it can be automatically and easily constructed from a UA. It uses only timers. Informally, if the alphabet of $\mathrm{A}^{\mathrm{ut}}$ is $\mathrm{V} \cup\left\{\right.$ tick\}, TimeA(A $\left.\mathrm{A}^{\mathrm{ut}}\right)$ is obtained as follows.

(a) $A^{u t}$ is projected into the alphabet $V$, for obtaining $\operatorname{Projv}\left(A^{u t}\right)$; (b) For each state $q$ of $\operatorname{Projv}\left(A^{u t}\right)$, several timers $\mathrm{Tq}_{\mathrm{i}}$ are defined, and their values tq $\mathrm{q}_{\mathrm{i}}$ are incremented at each tick.. 
(c) For each transition $\operatorname{Tr}$ of $\operatorname{Projv}\left(\mathrm{A}^{\mathrm{ut}}\right)$ which is executable from a state q1 and leads to a state q2 :

- Several enabling conditions $E_{i}\left(t_{q} 1_{j}\right)$ are defined. Each $E_{i}\left(t q 1_{j}\right)$ depends on a timer $T q 1_{i}$ of state $q 1$.

- Several initializations $\operatorname{tq}_{2} \leftarrow \mathrm{F}_{\mathrm{i}}\left(\mathrm{tq}_{\mathrm{i}}\right)$ are defined. Each initialization consists in initializing a

timer Tq2 $2_{j}$ of $q 2$ in function of the value tq $1_{i}$ of a timer Tq1 $1_{i}$ of $q 1$. The index $j$ also depends on tq $1_{i}$.

The semantics of the enabling conditions and of the initializations is the following.

- When a timer is initialized by an initialization, it becomes the active timer.

- The transition Tr may be executed only if its enabling condition depending of the active timer is true.

- When $\mathrm{Tr}$ is executed, a timer $\mathrm{Tq} 2_{\mathrm{j}}$ is initialized in function of the value of the current active timer. $\mathrm{Tq} 2_{\mathrm{j}}$ becomes the new active timer.

Let's give an idea of how the timers are defined. Each state $q$ of TimeA(Av) (and of Projv( $\left.A^{u t}\right)$ ) corresponds to a group $\mathrm{Gq}$ of states of $\mathrm{A}^{\mathrm{ut}}$ closed under the event tick. The group $\mathrm{Gq}$ may be composed by several sequences $\mathrm{Sq}_{\mathrm{i}}$ of states. Each $\mathrm{Sq}_{\mathrm{i}}$ contains a state, called first state, without ingoing tick, and all other states of $\mathrm{Sq}_{\mathrm{i}}$ are reachable from this first state by executing a few ticks. To each $\mathrm{Sq}_{\mathrm{i}}$, we associate a timer $\mathrm{Tq}_{\mathrm{i}}$ whose value is equal to zero in the first state of $\mathrm{Sq}_{\mathrm{i}}$. That is why several timers may be associated to each state of TimeA(Aut).

If we consider the untimed $\mathrm{A}^{\mathrm{ut}}$ of Figure 5.b, TimeA(A) $\left.\mathrm{A}^{\mathrm{ut}}\right)$ is represented on Figure 6. One timer T1 (resp. $\mathrm{T} 2$ ) is defined for state 1 (resp. state 2). For the transition from state 1 to state 2 : Its enabling condition is $\mathrm{E} 1(\mathrm{t} 1)=(\mathrm{t} 1 \leq 5) \wedge(\mathrm{t} 1>0)$, and its initialization is $\mathrm{t} 2 \leftarrow \mathrm{F} 1(\mathrm{t} 1)=\mathrm{t} 1-1$. For the two transitions from state 2 to state 1 : Their enabling condition is True, and their initialization is $\mathrm{t} 1 \leftarrow \mathrm{F} 2(\mathrm{t} 2)=0$.

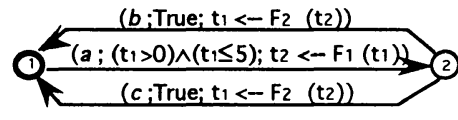

Figure 6. Temporized automaton

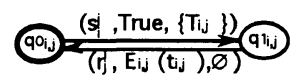

Figure 7. Supremal model SupMed $\mathrm{i}_{\mathrm{i} j}^{\mathrm{j}}$ of the medium for a pair $\left(\mathrm{PE}_{\mathbf{i}}, \mathrm{PE}_{\mathrm{j}}\right)$

We will see in the next section, that the untiming and timing operations may be convenient to resolve a real problem, such as designing real-time protocols.

6. Deriving protocols specifications providing real-time services

The desired service specification and the supremal model of the medium are the two starting points of the protocol derivation, and they are defined by timed automata (Sect. 3). .

\subsection{Supremal model of the medium}

Let's firstly remind that the supremal model of the medium is used in order to avoid the derivation of timing requirements impossible to respect by the medium (Sect. 2). The medium is assumed reliable and its supremal model is the following :

when $P E_{i}$ sends a message $m$ to $P E_{j}$, then the transit delay of $m$ in the medium belongs to an interval $I_{i, j}=\left[t_{i, j}^{\min } ; t_{i, j}^{\max }\right]$, where $t_{i, j}^{\min }$ and $t_{i, j}^{\max }$ are constant integers such that $1 \leq t_{i, j}^{\min } \leq t_{i, j}^{\max }<\infty$. Therefore, we suppose that there is at least one tick (Sect. 3) during the transmission of a message. For each pair $\left(\mathrm{PE}_{\mathrm{i}}, \mathrm{PE}_{\mathrm{j}}\right)$, this supremal model can be represented by a TA SupMed $\mathrm{i}_{\mathrm{i}, \mathrm{j}}$ represented on Figure 7.

For each pair $\left(\mathrm{PE}_{\mathbf{i}}, \mathrm{PE}_{\mathrm{j}}\right)$, the TA SupMed $\mathrm{i}_{\mathrm{i}, \mathrm{j}}$ (Fig.7) is defined by $\left(\mathrm{Q}_{\mathrm{i}, \mathrm{j}}, \mathrm{V}_{\mathrm{i}, \mathrm{j}},\left\{\mathrm{T}_{\mathrm{i}, \mathrm{j}}\right\},\left\{\mathrm{V}_{\mathrm{i}, \mathrm{j}}\right\}, \delta_{i, j}, \mathrm{q0} 0_{\mathrm{i}, \mathrm{j}}\right)$, where $Q_{i, j}=\left\{q 0_{i, j}, q 1_{i, j}\right\}, V_{i, j}=\left\{s_{i}^{j}, r_{j}^{j}\right\}, \delta_{i, j}=\left\{\left[q 0_{i, j}, s_{i}^{j}, q 1_{i, j}\right.\right.$, True,$\left\{T_{i, j}\right\}$, True $], \quad\left[q 1_{i, j}, r_{j}^{j}, q 0_{i, j}, E_{i, j}\left(t_{i, j}\right), \varnothing\right.$, True $\left.]\right\}$, with $E_{i, j}\left(t_{i, j}\right)=\left(t_{i, j}>\left(t_{i, j}^{\min }-1\right)\right) \wedge\left(t_{i, j} \leq t_{i, j}^{\max }\right)$.

SupMed $d_{i, j}^{t}$ uses one timer $T_{i, j}$ (Def.5) for defining timing requirements between $s_{i}^{j}$ and $r_{j}^{j}$, where the event $s_{i}^{j}$ means "PE $i$ sends a message to $P E_{j}$ ", and the event $r_{j}^{i}$ means "PE $E_{j}$ receives a message coming from $P E_{i}$ ". $A$ counter is not necessary, because timing requirements ensure the finiteness property (Def.1) due to $\left(t_{i, j}>\left(t_{i, j}^{\min }-1\right)\right)$ in the $T_{-}$Condition $E_{i, j}\left(t_{i, j}\right)$. Therefore, the $F_{-}$Condition is True.

Remark 9. Timing requirements on SupMed $\mathrm{d}_{\mathrm{i}, \mathrm{j}}^{\mathrm{a}}$ and $\operatorname{SupMed}_{\mathrm{j}, \mathrm{i}}^{\mathrm{t}}$ may be different.

6.2. Protocol derivation for sequential real-time systems 6.2.1. Service specification

The desired service is, with the supremal model of the medium, one of the two starting points of the 
protocol derivation. It is described by a TA, noted $S S^{t}$ and defined by $(Q, V, T,\{V\}, \delta, q 0)$, where $V$ is the set of interactions with the user, and $\mathrm{T}$ is the set of timers (Def.5) used for defining timing requirements between these interactions. Only one counter C, w.r.t. V (Def.8), is used, and the finiteness property (Def.1) is respected (Property 1). Informally, no more than Mc service primitives are executed during one unit of the global clock time (uct). Each event of $V$ is represented by $A_{i}$, where $A$ is the name of the primitive executed, and $\mathrm{i}$ identifies the protocol which executes $\mathrm{A}$.

Example 6. Here is a very simple service specified by $S S t=(Q, V, T,\{V\}, \delta, q 0)$ (Fig.8.a), with $Q=\{q 0, q 1\}$, $\mathrm{V}=\left\{\mathrm{A}_{1}, \mathrm{~B}_{2}\right\}, \mathrm{T}=\left\{\mathrm{T}_{1}\right\}, \delta=\left\{\left[\mathrm{q} 0, \mathrm{~A}_{1}, \mathrm{q} 1, \mathrm{E}\left(\mathrm{t}_{1}\right),\left\{\mathrm{T}_{1}\right\}, \mathrm{K}\left(\mathrm{c}_{1}\right)\right],\left[\mathrm{q} 1, \mathrm{~B}_{2}, \mathrm{q} 0, \mathrm{E}\left(\mathrm{t}_{1}\right),\left\{\mathrm{T}_{1}\right\}, \mathrm{K}\left(\mathrm{c}_{1}\right)\right]\right\}$, with $\mathrm{E}\left(\mathrm{t}_{1}\right)=\left(\mathrm{t}_{1} \leq 2\right)$, and $K\left(c_{1}\right)=\left(c_{1}<1\right)$. Informally, SSt uses one timer $T_{1}$ and one counter $C_{1}$, and specifies that : (a) events $A_{1}$ and $\mathrm{B}_{2}$ are executed alternatively; (b) at most one event occurs between two ticks (F_Condition $\mathrm{K}$ (c) c $\left._{1}\right)$; (c) at most two ticks occur between two consecutive events ( $\mathrm{T}_{-}$Condition $\mathrm{E}\left(\mathrm{t}_{1}\right)$ ). $\mathrm{SS} \mathrm{ut}_{=}=$UntimeA(SSt) (Def.26) is represented on Figure 8.b, and its states are $\left\langle\mathrm{q}, \mathrm{t}_{1}, \mathrm{c}_{1}\right\rangle$ where $\mathrm{q}$ is a state of $\mathrm{SSt}$.

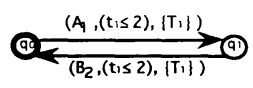

8.a. $S^{t}$

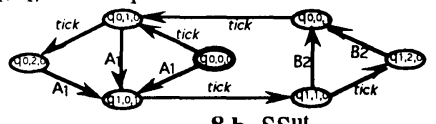

8.b. SSut

Figure 8. Timed and untimed sequential service specification

Definition 27. (outgoing, ingoing, out(q), in(q), outst $i(q)$, nbrout(q))

Let $S^{t}$ be a TA specifying a timed sequential service, and let $q$ be one of its states.

Outgoing (resp. ingoing) transitions of $\mathrm{q}$ are transitions which are executable from (resp. lead to) $\mathrm{q}$ out(q) (resp. in(q)) contains identifiers of SAP where outgoing (resp. ingoing) transitions of $q$ occur. outst $(q)$ is the set of states of $S^{t}$ reachable from $q$ by transitions executed by $P_{i}$. nbrout(q) is the number of transitions executable from $q$.

Example 7. for SSt of Example 6 (Figure 8.a), in $(q 0)=\{2\}, \operatorname{in}(q 1)=\{1\}$, out $(q 0)=\{1\}, \operatorname{out}(q 1)=\{2\}$, oust $_{1}(q 0)=\{q 1\}, \operatorname{oust}_{2}(q 0)=\varnothing$, oust $1(q 1)=\varnothing$, oust $2(q 1)=\{q 0\}, \operatorname{nbrout}(q 0)=\operatorname{nbrout}(q 1)=1$.

Since the starting points of the protocol derivation, i.e., the supremal model of the medium and the specification of the desired service, have been defined, we can propose a systematic method for deriving the specifications of : (a) the local protocol entities; (b) the necessary timing requirements on the medium, (Sect. 2). These derived specifications are first untimed automata (Def.26), and then are timed by using operator TimeA (Sect. 5).

\subsubsection{Transformation of the service specification}

The first thing to do is to transform SSt into another timed automaton TSSt (Transformed SSt) with the following rules.

First step : each timed transition of SSt:

$$
\text { is replaced by }
$$

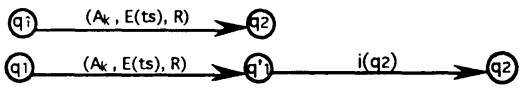

A new state $q^{\prime} 1$ is then inserted between each pair of states $q 1$ and $q 2$ connected by a transition. $\mathrm{q} 1$ and $\mathrm{q}^{\prime} 1$ are connected by an internal transition $\mathrm{i}(\mathrm{q} 2)$ parameterized by $\mathrm{q} 2$.

After this first step, we obtain a TA noted TSt. Let's notice that if a state of TSt is reachable by an internal transition $\mathrm{i}(\mathrm{q})$, then its outgoing transitions are not internal.

Second step : The specification TSt is transformed into an equivalent (Def.14) TSSt, such that every state q1 of TSS $^{t}$ respects either condition $\mathrm{C} 1$ or condition $\mathrm{C} 2$, defined below.

$\mathrm{C} 1=$ only an internal transition $\mathrm{i}(\mathrm{q})$ is executable from $\mathrm{q} 1$ (Fig. 9.a),

$\mathrm{C} 2$ = no internal transition is executable from q1, and all outgoing transitions (Def.27) of q1 are executable by a same protocol entity, i.e., cardinal of out(q1) is equal to one (|out(q1)|=1) (Fig.9.b). On Figure 9.b., out $(\mathrm{q} 1)=\{\mathrm{k}\}$ and $\operatorname{outst}_{\mathrm{k}}(\mathrm{q} 1)=\{\mathrm{r} 1, \ldots, \mathrm{rp}\}$.

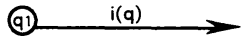

9.a. internal outgoing transition

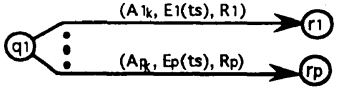

9.b. non internal outgoing transitions

Figure 9. Outgoing transitions in a state of the transformed specification TSSt. 
The way for obtaining TSS from TSt is the following. Every state $q$ of TS reachable by internal transition(s) (Fig.10.a), is replaced by as many states qi as the cardinal of out(q) (Fig. 10.b) . Outgoing transitions of states qi (which are not internal) must respect the preceding condition $\mathrm{C} 2$, and the following condition $\mathrm{C} 3$. Ingoing transitions of states qi must respect the following condition $\mathrm{C} 4$.

C3 : Outgoing transitions of two different states qi and qj of TSSt (Fig.10.b), generated from a same state q of TSt (Fig.10.a), are executed by two different protocol entities.

C4 : The sets of ingoing transitions (which are internal) of two different states qi and qj of TSS , generated from a same state q of TSt, are equal to the set of ingoing transitions of state q (Fig.10).

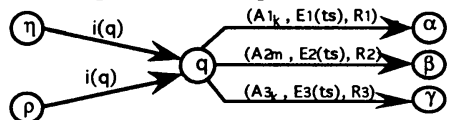

10.a. State e in TSt

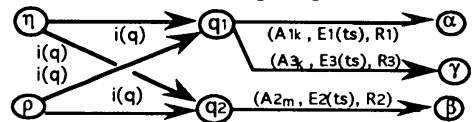

10.b. Transformation of $e$ in TSS

Figure 10. Example of transformation from TSt to TSS ${ }^{t}$

Remark 10. (a) if two states $\mathrm{r} 1$ and $\mathrm{r} 2$ of TSSt are connected by a transition $\mathrm{i}(\mathrm{q})$ then $\operatorname{lin}(\mathrm{r} 1)|=| \operatorname{out}(\mathrm{r} 2) \mid=1$;

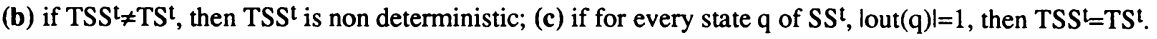

Definition 28. (Operator Transf) Operator Transf is simply defined by : $\mathbf{T S S} \mathbf{t}=\operatorname{Transf}(\mathrm{SS}$ ).

Example 8. SSt of Example 6 (Fig.8.a.) is transformed into TSSt of Figure 11. In this example, only the first step of the transformation is used, because lout(q0)|=|out(q1)|=1 (Remark 10.c).

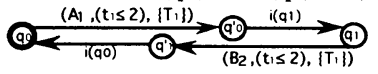

Figure 11. Transformation of $S S^{t}$ of Figure 8.a.

\subsubsection{Procedure of protocole derivation for a sequential system}

Considering a TA SSt (Sect.6.2.1), and a TA SupMed $\mathrm{d}_{\mathrm{i}, \mathrm{j}}\left(\mathrm{Sect.6.1}^{\mathrm{t}}\right.$ ) for each pair $\left(\mathrm{PE}_{\mathrm{i}}, \mathrm{PE}_{\mathrm{j}}\right)$, the proposed procedure of protocol derivation, is called Der_Seq_Prot and consists of eight steps.

Step 1 : SSt is transformed into TSSt, i.e., TSS $t=$ Transf $\left(\mathrm{SS}^{t}\right)$ (Sect.6.2.2, Def.28).

Step 2 : From TSSt and the different SupMed ${ }_{i, j}^{t}$, we generate $\operatorname{MedSS}_{\varepsilon}^{t}$ with the following rules :

- A not internal transition remains unchanged.

- An internal transition $\mathrm{i}(\mathrm{q}) \quad$ (1) $\mathrm{i}(\mathrm{q}) \longrightarrow$ (a)

Case $a:$ if in(q1)=out(q2) (def.28), the transition becomes : (a1)

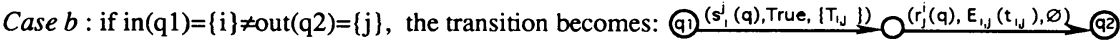

This transformation uses SupMed $d_{i, j}$ (Sect.6.1), but with $s_{i}^{j}$ and $r_{j}^{i}$, (Sect.6.1) parameterized by q. Informally, $i(q)$ consists in : (a) doing nothing, if it connects two consecutive transitions of SSt executed by a same $P E_{i}$; (b) sending a message from $P E_{i}$ to $P E_{j}$, if it connects two consecutive transitions of $S S^{t}$ respectively executed by $P E_{i}$ and $P E_{j}$. The message is parameterized by $q$.

Step 3 : Transitions $\varepsilon$ of MedSS $_{\varepsilon}^{t}$ are removed by projection for obtaining MedSSt. An algorithm for removing these $\varepsilon$ is proposed in [BC79].

Step 4 : MedSSt is untimed (Def.26) for obtaining MedSS ${ }^{\mathrm{t}}=$ UntimeA(MedSS $^{\mathrm{t}}$ ). MedSS ${ }^{\mathrm{ut}}$ is a minimal FSM containing the event tick. Let's notice that the three remaining steps process FSMs with event $t i c k$.

Step 5 : we generate an untimed automaton GPS ${ }^{\text {ut }}$ (global protocol specification), by adding a second parameter to each event $s_{i}^{j}(q)$ or $r_{j}^{j}(q)$ in MedSS ${ }^{u t}$, with the following rule :

A transition (a) $s_{i}^{j}(q) \longrightarrow$ (q2) is replaced by a transition (91) $s_{i}^{j}(q, q 2) \rightarrow$ (q2). The same transformation is made on transitions $r_{j}^{i}(q)$. This transformation allows to differentiate two transitions $s_{i}^{j}(q)$ (or $\mathrm{r}_{\mathrm{j}}^{\mathrm{j}}(\mathrm{q})$ ) which do not lead to the same state in MedSSut.

Informally, a message is sent from a $P E_{i}$ with two parameters $q$ and $q 2$ (event $s_{i}^{j}(q, q 2)$ ), and may be 
received by a $P E_{j}$ with a different second parameter $q^{\prime 2}$ (event $r_{j}^{j}\left(q, q^{\prime} 2\right)$ ). This means that the medium not only carries messages, but it also modifies their second parameters. This modification informs the receiving protocol entity about the transit delay of the message in the medium.

Step 6 :For each $P E_{i}$, the untimed automaton PS $_{i}^{u t}$ is derived by projecting GPSut in the alphabet $V_{i} \cup\left\{\right.$ tick\}, where $V_{i}$ contains all events in GPSut executed by $P E_{i}$. An event of $V_{i}$ may correspond to :

(a) execution of a primitive by $P E_{i} ;$ (b) an event $s_{i}^{j}(q, q 2)$; (c) an event $r_{i}^{k}(q, q 2)$, with $j, k \neq i$.

Step 7 : For each pair $\left(P E_{i}, P E_{j}\right)$ and each $q$, where $P E_{i}$ sends to $P E_{j}$ a message whose first parameter is $q$ (i.e., events $s_{i}^{j}(q, *)$ and $r_{j}^{j}(q, *)$ exist in GPS $\left.{ }^{u t}\right)$, the untimed automaton $\operatorname{ReqMed}_{i, j}^{\mathrm{ut}}(q)$ is generated by projecting GPSut in the alphabet $V_{i, j}(q) \cup\{$ tick $\}$. An element of $V_{i, j}(q)$ may be any event $s_{i}^{j}(q, *)$ and $\mathrm{r}_{\mathrm{j}}^{\mathrm{j}}(\mathrm{q}, *)$ of GPSut. The obtained ReqMed $\mathrm{d}_{\mathrm{i}, \mathrm{j}}^{\mathrm{ut}}(\mathrm{q})$ specifies the behaviour of the medium when it carries, from $P E_{i}$ to $P E_{j}$, a message whose first parameter is $q$.

The informal semantics of the different $P_{i}^{u t}\left(\operatorname{Step~6)}\right.$ and $\operatorname{ReqMed}_{i, j}^{\mathrm{ut}}(\mathrm{q})$ (Step 7) is the following. Let $n$ be the number of protocol entities $P E_{i}$, for $i=1, \ldots, n$. If $P E_{i}$ are specified by $P S_{i}^{u t}$, and if the medium respects the specifications $\operatorname{ReqMed}_{\mathrm{i}, \mathrm{j}}^{\mathrm{ut}}(\mathrm{q})$, then the service $S S^{t}$ is totally or partially provided (Def.29 and 30).

Step 8 : The untimed specifications $\mathrm{PS}_{\mathrm{i}}^{\mathrm{ut}}$ and $\operatorname{ReqMed}_{\mathrm{i}, \mathrm{j}}^{\mathrm{tt}}(\mathrm{q})$ obtained at Steps 6 and 7 are timed, by using the operator TimeA (Sect.5).

End of Der_Seq_Prot

Remark 11. (a) In Steps $1,2,3$, SS $^{t}, \mathrm{TSS}^{\mathrm{t}}, \mathrm{MedSS}_{\varepsilon}^{\mathrm{t}}$, and MedSSt use the same counter C1, w.r.t. the alphabet V of SSt; (b) MedSS $S_{\varepsilon}^{t}$ and MedSSt use timers of $S S^{t}$ and a timer $T_{i, j}$ for each pair $\left(P_{i}, P E_{j}\right)$ where $P E_{i}$ sends a message to $P E_{j}$.

Definition 29. (Provided service PrSSut) For obtaining an untimed automaton (with event tick) specifying the service provided to the user, and noted PrSSut, one only has to project MedSSut (Step 4) in V $\cup\{$ tick\}, where $\mathrm{V}$ is the alphabet of SSt. Informally, this projection consists in keeping visible, in sequences accepted by MedSSut, only events of SSut.

Definition 30. (Service totally or partially provided)

Let SSut and PrSSut be untimed automata specifying respectively the desired and the provided service. The service is said totally provided $\quad$ if and only if : $\operatorname{PrSS} \mathrm{ut} \cong S S^{u t}$, i.e., $\mathcal{L}_{\mathrm{PrSSut}}=\mathcal{L}_{\mathrm{SS}}$, The service is said partially provided if and only if : PrSSut $<S S u t$, i.e., $\mathcal{L}_{\mathrm{PrSS}} \subset \mathcal{L}_{\mathrm{SS}}$ ut .

Theorem 6. If SSt specifies a desired service, let $S S^{u t}=U n t i m e A\left(S S^{t}\right)(D e f .26)$, and let PrSS ut be the specification of the provided service (def.30). Then: PrSSut $\leq$ SSut (i.e., $\mathcal{L}_{\text {PrSSut }} \subseteq \mathcal{L}_{\text {SSut }}$ ).

The safety is then ensured.

\subsubsection{Example}

We consider the SSt of Example 6 (Fig.8.a), with $\mathrm{q} 0=1$ and $\mathrm{q} 1=2$. The supremal model of the medium is defined by SupMed $d_{1,2}$ and SupMed $\mathbb{t}_{2,1}$ (Sect.6.1, Fig.7), with $t_{1,2}^{\min }=t_{2,1}^{\min }=1$ and $t_{1,2}^{\max }=t_{2,1}^{\max }=2$. Informally, during the transmission of a message, one or two ticks of the global clock may occur.

By using the procedure Der_Seq_Prot (Sect.6.2.3), we obtain, after the seventh step, the specifications $\mathrm{PS}_{1}^{\mathrm{ut}}$ and $\mathrm{PS}_{2}^{\mathrm{ut}}$ of Figure 12, and $\operatorname{ReqMed}_{1,2}^{\mathrm{ut}}(2)$ and $\operatorname{ReqMed}_{2,1}^{\mathrm{ut}}(1)$ of Figure 13.

By using the operator TimeA (Sect. 5), we obtain the temporized automata represented on Figure 14.

As an example, TimeA(PS ${ }_{1}^{\text {ut }}$ ) (Fig. 14.a) is detailed below.

TimeA(PS 1 ) : Timers $\mathrm{T} 11$ and T12 are respectively associated to states 1 and 2 .

Timers $\mathrm{T} 13_{1}$ and $\mathrm{T} 13_{2}$ are associated to state3.

Transition $\operatorname{Tr} 11$ : Event $a ; \quad$ EC $: \mathrm{t} 11 \leq 2$; In $:$ t12 $\leftarrow 0$.

Transition Tr12 : Event $s_{1}^{2}(2,3)$; EC : $(t 12=0)$; In : $\mathrm{t} 13_{1} \leftarrow 0$.

Transition $\operatorname{Tr} 13$ : Event $\mathrm{s}_{1}^{2}(2,7)$; EC : $(\mathrm{t} 12=1)$; In $: \mathrm{t} 13_{2} \leftarrow 0$. 
Transition Tr14 : Event $r_{1}^{2}(1,2) ;$ ECs : $\left(t 13_{1}>1\right) \wedge\left(t 13_{1} \leq 3\right),\left(t 13_{2}=1\right) ;-\cdots-\cdots---$ In : $t 11 \leftarrow 1$. Transition $\operatorname{Tr} 15$ : Event $r_{1}^{2}(1,5)$; EC: $\left(t 13_{1}>2\right) \wedge\left(t 13_{1} \leq 4\right),\left(t 13_{2}=3\right) ;-\ldots-\ldots-\ldots . .-$ In : $t 11 \leftarrow 2$.

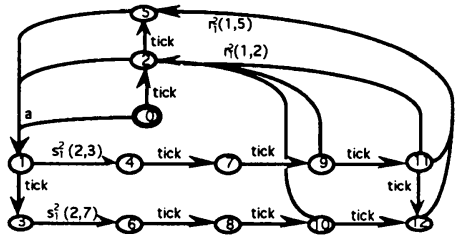

12.a. $P S_{1}^{\text {ut }}$

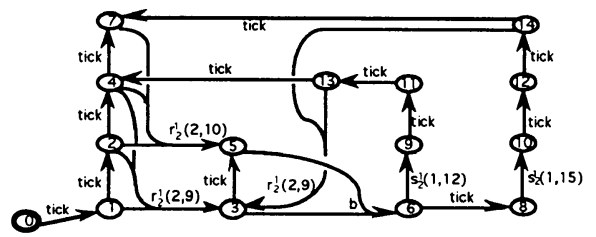

12.b. $\mathrm{PS}_{2}^{\mathrm{ut}}$

Figure 12. Obtained untimed protocol

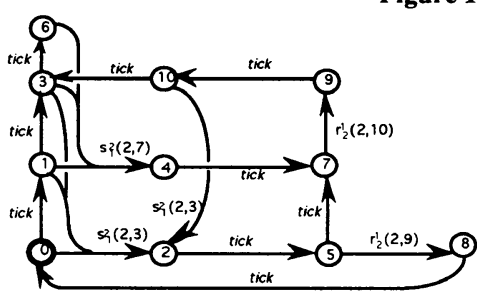

13.a. $\operatorname{ReqMed} \mathrm{ut}_{1,2}^{\mathrm{ut}}(2)$

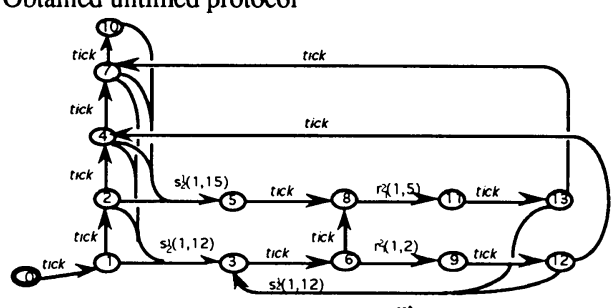

13.b. $\operatorname{ReqMed}_{2,1}^{\mathrm{ut}}(1)$

Figure 13. Obtained untimed medium

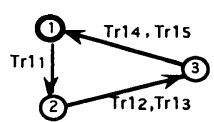

14.a. TimeA(PS $\left.{ }_{1}^{\mathrm{ut}}\right)$

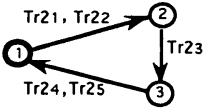

14.b. Time $A\left(\mathrm{PS}_{2}^{\mathrm{ut}}\right)$ 14.c. $T$ ime $A\left(\operatorname{ReqMed}_{1,2}^{\mathrm{ut}}(2)\right)$ 14.d. $T i m e A\left(\operatorname{ReqMed}_{2,1}^{\mathrm{ut}}(1)\right)$<smiles></smiles>

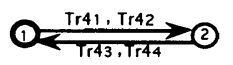

Figure 14. Obtained timed specifications

\subsection{Protocol derivation for parallel and concurrent real-time systems}

\subsubsection{Introduction}

For the sake of simplicity, we only consider a parallel system composed by only two sequential systems. A desired parallel service is then specified by two TA (Def.13) SSt $[i]$ over alphabets V[i], for $i=1,2$. Each SSt $[i]$ specifies a sequential desired service. Let's consider three cases :

a) $V[1] \subseteq V[2]: S^{t}=S S^{t}[1] \otimes S S^{t}[2]$ (Def.21) is a sequential service (Remark 3.b), and we may use the procedure Der_Seq_Prot (sect.6.2.3) for deriving the protocol providing the service specified by SSt.

b) $\mathrm{V}[\mathrm{i}] \neq \varnothing$ and $\mathrm{V}[\mathrm{i}] \cap \mathrm{V}[\mathrm{j}]=\varnothing$, for $\mathrm{i}, \mathrm{j}=1,2$, and $\mathrm{i} \neq \mathrm{j}: \mathrm{SS}^{\mathrm{t}}[1]$ and $\mathrm{SS}^{\mathrm{t}}[2]$ are independent and compose a parallel system (Def.23). We may process each sequential service separately, i.e., for each SSt[i], we use Der_Seq_Prot for deriving the sequential protocol which provide SSt $[\mathrm{i}]$.

c) $\mathrm{V}[\mathrm{i}]-\mathrm{V}[\mathrm{j}] \neq \varnothing$ and $\mathrm{V}[\mathrm{i}] \cap \mathrm{V}[\mathrm{j}] \neq \varnothing$, for $\mathrm{i}, \mathrm{j}=1,2$, and $\mathrm{i} \neq \mathrm{j}$ : $\mathrm{SS}^{\mathrm{t}}[1]$ and $\mathrm{SS}^{\mathrm{t}}[2]$ are dependent and compose a concurrent system (Def.23). This case is studied in detail in the present Section 6.3.

\subsubsection{Solution for the problem of the choice}

In a concurrent system, we think that one of the main problems consists in avoiding possible deadlocks. For that, we use the following approach, already proposed in [KHB92] :

During the running of a concurrent distributed system, when a choice is possible between execution of different transitions, this choice is made locally by a same protocol entity.

Let $P E_{i}$, for $i=1, \ldots, n$, be the $n$ protocol entities which execute the transitions of $S^{2}[1]$ and $S S^{t}[2]$. For the sake of simplicity, we suppose that all choices are made by a same protocol entity $\mathrm{PE}_{\mathrm{c}}$, with $\mathrm{c}>\mathrm{n}$. In other words, if there is a choice to make, the protocol entities $\mathrm{PE}_{\mathrm{i}}$, for $\mathrm{i}=1, \ldots, n$, "pass the buck" to $\mathrm{PE}_{\mathrm{c}}$. Such constraint seems too restrictive, and we intend to weaken it in a next version. To enforce explicitly this 
choice, we must add to SSt[1] and SSt[2], some timed events (Def.11) noted ( $\mathrm{i}_{\mathrm{c}}$, True, $\varnothing$, True), where $\mathrm{i}_{\mathrm{c}}$ is executed by $P E_{c}$. These timed events are added as follows : for each state $q$ of SSt $\left.i\right]$, for $i=1,2$, where nbrout(q) $>1$ (Def.27), its ougoing transitions $\operatorname{Tr}_{1}, \ldots, \operatorname{Tr}_{m}$ represented in Figure 15.a. are replaced by the structure of Figure 15.b. The obtained specifications are noted $S S_{c}^{t}[1]$ and $S S_{c}^{t}[2]$. Let's now propose a procedure of protocol derivation for concurrent systems.

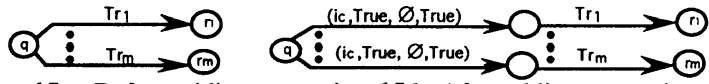

15.a. Before adding events $i_{c} \quad$ 15.b. After adding events $i_{c}$

Figure 15. Adding events $i_{c}$

6.3.3. Procedure of protocol derivation for a concurrent system

Let two TA SSt[i] over alphabets V[i], for $i=1,2$, and a TA SupMed $\mathrm{u}_{\mathrm{u}, \mathrm{v}}$ for each pair $\left(\mathrm{PE}_{\mathrm{u}}, \mathrm{PE}_{\mathrm{v}}\right)$, the procedure of protocol derivation for concurrent systems, called Der_Conc_Prot, consists of nine steps.

Step one : $S S t[i]$ are modified into $S_{c}^{t}[i]$, for $i=1,2$, (sect.6.3.2.). Besides, any two states of respectively $S S_{c}^{t}[1]$ and $S S_{c}^{t}[2]$ must be identified differently. This is necessary for not confusing exchanged messages, which are parameterized by identifiers of states (see Der_Seq_Prot in Sect.6.2.3).

Step two : Steps 1 to 5 of Der_Seq_Prot are applied to each $S_{c}^{t}[i]$ for obtaining GPS ${ }_{c}^{u t}[i]$, for i=1,2, but with the following difference. At the third step of Der_Seq_Prot, not only transitions $\varepsilon$, but also transitions $\left(\mathrm{i}_{\mathrm{c}}\right.$, True,$\left.\varnothing\right)$, are removed. Let $\mathrm{V}_{\mathrm{g}}[\mathrm{i}] \cup\{$ tick $\}$ be the alphabet of GPS $\mathrm{c}_{\mathrm{c}}^{\mathrm{ut}}[\mathrm{i}]$, then $\mathrm{V}[\mathrm{i}] \subseteq \mathrm{V}_{\mathrm{g}}[\mathrm{i}]$.

Step three : The synchronized product $\mathrm{GPS}_{c}^{\mathrm{ut}}=\mathrm{GPS}_{c}^{\mathrm{ut}}[\mathrm{i}] \times \mathrm{GPS}_{c}^{\mathrm{ut}}[\mathrm{i}]$ is computed (Remark 7.b).

Step four : Indesirable states are removed from GPS $\mathrm{c}_{\mathrm{c}}^{\mathrm{ut}}$ for obtaining GPSut. A state is indesirable if it is either a deadlock or only a selfloop tick is executable from it (Remark 6). For removing indesirable states, we may use a fixpoint method similar to the one used in the control theory for computing controllable languages ([WR87,KBD94]).

Step five : The protocol specification $\mathrm{PS}_{\mathrm{c}}^{\mathrm{ut}}$ of $\mathrm{PE}_{\mathrm{c}}$ (Sect.6.3.2) is obtained by projecting GPSut in alphabet $\mathrm{V}_{\mathrm{c}} \cup\{$ tick $\} . \mathrm{V}_{\mathrm{c}}$ contains all events of GPSut executed by $P E_{\mathrm{c}}$, and these events are of the form $\mathrm{s}_{\mathrm{c}}^{*}(*, *)$ and $r_{c}^{*}(*, *)$ (see Sect.6.1, and Step two of Der_Seq_Prot), where * may be any parameter.

Step six : the sequential GPS ${ }^{u t}[i]$ are obtained by projecting GPSut in alphabets $V_{g}[i] \cup\{t i c k\}$ of GPS ${ }_{c}^{u t}[i]$, (Step 2), for $i=1,2$. The sequential processes specified by GPS ${ }_{c}^{u t}[i]$, for $i=1,2$, interact with $P_{c}$ specified by $\mathrm{PS}_{\mathrm{c}}^{\mathrm{ut}}$ and do not lead to an indesirable state.

Step seven : For each GPSut[i] (for i=1,2), we apply Step 6 of Der_Seq_Prot for obtaining the untimed automata (UA) $P S_{j}^{\mathrm{ut}}[\mathrm{i}]$ corresponding to $P E_{\mathrm{j}}(\mathrm{j}=1, \ldots, \mathrm{n})$.

Step eight : For each GPSut[i] (for $\mathrm{i}=1,2$ ), we apply Step 7 of Der_Seq_Prot for obtaining the UA $\operatorname{ReqMed}_{j, k}^{\mathrm{ut}}(q)$. Each ReqMed $\mathrm{R}_{j, k}^{\mathrm{ut}}(q)$ depends implicitly on $\mathrm{i}$, because $q$ identifies a state of $S_{\mathrm{c}}^{t}[i]$, and states of $S_{c}^{t}[1]$ and $S_{c}^{t}[2]$ are identified differently (see Step one).

The informal semantics of PS $\mathrm{c}_{c}^{\mathrm{ut}}$ (Step 5), of PS $\mathrm{j}_{\mathrm{j}}^{\mathrm{ut}}\left[\mathrm{i}\right.$ (Step 7), and of ReqMed $\mathrm{d}_{\mathrm{j}, \mathrm{k}}^{\mathrm{ut}}(\mathrm{q})$ (Step 8) is the following. If each $\mathrm{PE}_{\mathrm{j}}$, for $\mathrm{j}=1, \ldots, n$, is a parallel system specified by two $\mathrm{PS}_{\mathrm{j}}^{\mathrm{ut}}[\mathrm{i}]$, for $\mathrm{i}=1,2$, and if the medium respects the specifications $\operatorname{ReqMed}_{\mathrm{i}, \mathrm{j}}^{\mathrm{ut}}(q)$, then the desired concurrent service specified by SSt[1] and SSt[2] (step one), is totally or partially provided by the help of $\mathrm{PE}_{\mathrm{c}}$ specified by $\mathrm{PS}_{\mathrm{c}}^{\mathrm{ut}}(\mathrm{Step} 5$ ).

Step nine : The untimed specifications obtained at Steps 5, 7 and 8 are timed, by using the operator TimeA (Sect.5).

End of Der_Conc_Prot

\section{Conclusion}

In this paper, we present a model we have developed for specifying real-time discrete event systems. An application of the model for designing real-time protocols is also proposed. The synthesis approach used for deriving a real-time protocol providing a desired service is inspired by other works, but our main contribution has been to consider real-time systems, i.e., systems containing timing requirements. We conclude this study by making an informal and succint comparison between our model for specifying 
DESs, and the two models which have mainly inspired us. A few extensions are also proposed.

First model ([Os90, BW92, OW90]) : For defining a TA At, a global clock and a set of timers are used. For each transition $\mathrm{Tr}_{\mathrm{i}}$ of $\mathrm{A}^{t}$ corresponds one timer $\mathrm{T}_{\mathrm{i}}$. This timer is reset only when a state q1, from which $\mathrm{Tr}_{\mathrm{i}}$ is executable, is reached. This same timer is not used in another state $\mathrm{q} 2 \neq \mathrm{q} 1$. The enabling condition of $\mathrm{Tr}_{\mathrm{i}}$ is that the value of $\mathrm{T}_{\mathrm{i}}$ must belong to an interval. We think that our model is more general because (in our model): (a) the enabling condition of a transition may depend on several timers; (b) a same timer may be used in the enabling conditions of several transitions; (c) a timer may be reset at the occurrence of any transition; (d) the finiteness property may be ensured by using counters; (e) the operator TimeA is defined.

Second model ([AD90, TH92]) : For defining a timed automata, a dense time and a set of clocks are used. These clocks are used as we use the timers in our model, but the semantics is quite different, because the clocks are not synchronized. Besides : (a) Our operators UntimeA and TimeA are different than operators Untime and Time proposed in [TH92]. In fact in [TH92], from a TA At, the operator Untime is used for

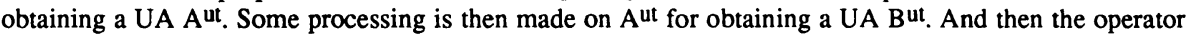
Time can be applied on $\mathrm{B}^{\mathrm{ut}}$ only if the processing for obtaining $\mathrm{B}^{\mathrm{ut}}$ from $\mathrm{A}^{\mathrm{ut}}$ makes no projection, i.e., $\mathrm{A}^{\mathrm{ut}}$ and $B^{u t}$ have a same alphabet; (b) The finiteness property is supposed respected in [TH92], but it is not ensured. Another advantage with our model is then that we can ensure the finiteness property. (c) In [TH92] the composition is defined only when the two TA have a same alphabet, and in [AD90], authors only specify how events executed conjointly by the two composed systems are processed. Our limitation is that the time is discrete.

Extensions : The exponential complexity in the number of timers imposes to investigate how to choose classes of systems which avoid this computational blow-up. The simplest but also the most restrictive class contains systems respecting the following condition : timing requirements are only between consecutive events. With such systems, only one timer is necessary, therefore the complexity becomes polynomial. In the presented model, the enabling conditions use only operators $\leq,>$ and $=$ for defining canonical boolean functions. We are also investigating how we can modify systematically several existing protocol entities, which provide an old service, for providing a new desired service. For that, we intend to use control theory of the discrete event systems .

\section{REFERENCES}

[AD90] R. ALur and D.Dill, "Automata for Modeling Real-Time Systems." In Lecture Notes in Computer Science 443, editor Proceedings of the 17th Intern. Coll. on Automata, Languages and Programming, Warwick, UK, 1990. Springer-Verlag.

[BC79] W.A.Barrett and J.D.Couch,"Compiler Construction:Theory and Practice" Ed.:Science Research Associates, Inc. 1979

[BG86] G.v. Bochmann and R. Gotzhein, "Deriving protocols specifications from service specifications." Proceedings du Symposium ACM SIGCOM' 86, Vermont, USA, pp.148-156, 1986.

[BW92] B. Brandin and W.M. Wonham, "The supervisory Control of Timed Discrete-Event Systems." Proceedings of the 31rst Conf. on Decision and Control, Tucson, Arizona, Dec.92.

[Di89] D. Dill, "Timing assumptions and Verifications of Finite-State Concurrent Systems.", In Lecture Notes in Computer Sciences 407, editor Automatic Verification Methods For Finite State Systems, Intern. Workshop, pp.197-212, Grenoble France, 1989. Springer-Verlag.

[KBD93] A. Khoumsi, G.v. Bochmann and R. Dssouli, "Dérivation de spécifications de protocoles à partir de spécifications de services avec contraintes temporelles." Colloque Francophone pour l'ingénierie des protocoles (CFIP), Montreal, September 1993.

[KBD94] A. Khoumsi, G.v. Bochmann and R. Dssouli, "Contrôle et extension des systèmes à événements discrets totalement et partiellement observables." Third Maghrebian Conference on Software Engineering and Artificial Intelligence, Rabat, April 1994.

[KBK89] F. Khendek, G.v. Bochmann and C. Kant, "New results on deriving protocol specifications from services specifications", Proceedings of the ACM SIGCOMM'89, pp.136-145, 1989.

[KHB92] C. Kant, T. Higashino and G.v. Bochmann, "Deriving protocol specifications from service specifications written in LOTOS." Rapport interne No 805, Département d'Informatique et de Recherche Opérationnelle. Faculté des arts et des sciences, Université de Montréal, January 1992.

[Os90] J.S. Ostroff, "Deciding Properties of Timed Transitions Models." IEEE Transactions on Parallel and Distributed Systems, Vol.1, No.2, pp.170-183, April 1990.

[OW90] J.S. Ostroff and W.M. Wonham, "A framework for real-time discrete event control." IEEE Transactions on Automatic Control, Vol.35, No.4, pp.386-397, April 1990.

[SP90] K.Saleh and R. Probert, "A service-based method for the synthesis of Communications protocols." International Journal of Mini and Microcomputers, Vol.12, No 3, 1990.

[TH92] H.W-Toi and Gérard Hoffmann, "The Control of Dense Real-Time Discrete Event Systems." Report submitted to IEEE Transactions on Automatic Control, February 1992.

[WR87] W.M. Wonham and P.J. Ramadge, "On the Supremal Controllable sublanguage of a Given Language." SIAM J.Control and Optimization, Vol.25, No.3, May 1987. 